\title{
Rohstoffsicherung in der Landes- und Regionalplanung
}

\author{
Andrea Hartz ${ }^{1} \cdot$ Sascha Saad ${ }^{1}$ - Lydia Schniedermeier ${ }^{1} \cdot$ Mark Fleischhauer $^{2} \cdot$ Stefan Greiving $^{2}$
}

Eingegangen: 29. September 2017 / Angenommen: 25. April 2018 / Online publiziert: 18. Mai 2018

(c) Springer-Verlag GmbH Deutschland, ein Teil von Springer Nature 2018

\section{Zusammenfassung}

Mineralische Rohstoffe finden in nahezu allen Produktionszweigen Verwendung. Die Rohstoffgewinnung steht dabei in einem Wettbewerb mit anderen Flächennutzungen und trifft vielerorts auf wenig Akzeptanz. Der Raumordnung kommt eine wichtige Rolle bei der Flächenvorsorge für die Rohstoffgewinnung wie auch bei der langfristigen Sicherung von Rohstoffpotenzialen zu. Im Rahmen der Raumordnung erfolgt die Abwägung mit anderen Raumnutzungen und Raumfunktionen im Sinne einer nachhaltigen Raumentwicklung. Der Beitrag beschäftigt sich mit der Frage, wie die Landes- und Regionalplanung dieser Aufgabe in Bezug auf nichtenergetische mineralische Rohstoffe nachkommt. Die Ergebnisse von Befragungen und Plananalysen zeigen, dass der Regelungsumfang zur Rohstoffsicherung in den Planwerken der Länder und Regionen teils sehr deutlich variiert. Darauf aufbauend werden Lösungsansätze für eine angemessene Berücksichtigung der Rohstoffsicherung in der Landes- und Regionalplanung aufgezeigt. Diese beziehen sich sowohl auf die methodischen Herausforderungen im Zuge der Abwägung als auch auf die Ausgestaltung des raumordnerischen Instrumentariums.

Schlüsselwörter Mineralische Rohstoffe $\cdot$ Raumordnung $\cdot$ Rohstoffabbau $\cdot$ Rohstoffsicherung $\cdot$ Folgenutzungen

Andrea Hartz

andreahartz@agl-online.de

Sascha Saad

saschasaad@agl-online.de

Lydia Schniedermeier

lydiaschniedermeier@agl-online.de

Dr. Mark Fleischhauer

fleischhauer@plan-risk-consult.de

Prof. Dr. Stefan Greiving

stefan.greiving@tu-dortmund.de

1 agl Hartz $\cdot$ Saad $\cdot$ Wendl I Landschafts-, Stadtund Raumplanung, Großherzog-Friedrich-Straße 16-18, 66111 Saarbrücken, Deutschland

2 plan + risk consult - Prof. Greiving und Partner, Stockumer Straße 435/437, 44227 Dortmund, Deutschland 


\title{
Securing of mineral resources in spatial and regional planning
}

\begin{abstract}
Mineral resources are used in virtually all branches of production. The extraction of resources is competing with other land uses and often meets little acceptance. Spatial planning plays an important role not only for precautionary land use for resource extraction, but also for long-term securing of resource potentials. The spatial planning involves a weighing against other land uses and functions in the sense of a sustainable regional development. This paper looks into the question how the statewide and regional planning meets this requirement with regard to non-energetic mineral resources. The results of polls and plan analyses show massive variations in the regulatory scope for securing resources in the statewide and regional plans. This serves as a basis on which solutions are highlighted for an adequate consideration of securing resources in spatial planning. These relate to the methodical challenges in the weighing process as well as the design of the spatial planning toolsets.
\end{abstract}

Keywords Mineral resources $\cdot$ Spatial planning $\cdot$ Resource extraction $\cdot$ Securing resources $\cdot$ Subsequent uses

\section{Einleitung}

Der Beitrag befasst sich mit den raumordnerischen Steuerungsaufgaben und -optionen bei der Rohstoffgewinnung und der langfristigen Sicherung nichtenergetischer mineralischer Rohstoffe. Es werden die Rahmenbedingungen für Rohstoffabbau und -sicherung in Deutschland dargelegt und beleuchtet, welche Rolle die Rohstoffsicherung als Handlungsfeld der Raumordnung im Spannungsfeld unterschiedlicher und teilweise widerstreitender Interessen einnimmt. Auf der Basis umfangreicher Analysen von Regionalplänen und Landesraumordnungsplänen sowie Befragungen wird ein Überblick über den aktuellen Stand der raumordnerischen Rohstoffsicherung gegeben und anhand von Beispielen konkretisiert. Der Schwerpunkt des Beitrags liegt auf der Ausgestaltung der raumordnerischen Abwägung wie auch des raumordnerischen Instrumentariums. Der Beitrag basiert auf folgenden Forschungsfragen:

- Welche Herausforderungen ergeben sich für die Raumordnung bei der Rohstoffsicherung auf Länder- und regionaler Ebene?

- Welche Grundlagen sind für eine fachgerechte raumordnerische Abwägung der Rohstoffsicherung mit anderen Belangen erforderlich?

- Welche methodischen Vorgehensweisen eignen sich für eine raumordnerische Abwägung? Wie können Vorbelastung und kumulative Effekte sinnvoll in die Abwägung integriert werden?

- Welche raumordnerischen Instrumente lassen sich für eine räumliche und zeitliche Steuerung der Rohstoffgewinnung und Rohstoffsicherung einsetzen?

- Wie können weitere Aspekte, wie beispielsweise Zwischen- und Folgenutzungen, in die Regionalplanung eingebettet werden? Welche informellen Strategien, Konzepte, Verfahren und welche dialogischen Prozesse kön- nen die formalen Planungen sinnvoll vorbereiten, flankieren oder in die Umsetzung bringen?

Der Beitrag nimmt Bezug auf die Ergebnisse des Modellvorhabens der Raumordnung (MORO) „Mittel- und langfristige Sicherung mineralischer Rohstoffe in der landesweiten Raumplanung und in der Regionalplanung“" (BMVI 2017).

Zunächst erfolgt ein Überblick über die Situation der mineralischen Rohstoffe in Deutschland (Kapitel 2). Beleuchtet werden zudem das raumordnungspolitische Handlungsfeld (Kapitel 3) sowie der aktuelle Stand der Rohstoffsicherung in der Raumordnung (Kapitel 4). Darauf aufbauend werden konzeptionelle Überlegungen zur Rohstoffsicherung in der Raumordnung dargelegt (Kapitel 5) und die wichtigsten Erkenntnisse in einem Fazit zusammengefasst (Kapitel 6).

\section{Mineralische Rohstoffe in Deutschland - ein Überblick}

\subsection{Lagerstätten, Fördermengen und Flächenansprüche}

Nichtenergetische mineralische Rohstoffe umfassen Metallrohstoffe (Erze), Industriemineralien sowie Steine und Erden (Krumm 2014: 3). Sie finden in nahezu allen Produktionszweigen Verwendung, unter anderem im Bauwesen, in der chemischen Industrie, in der Automobilindustrie und der Mikroelektronik. Die Verfügbarkeit mineralischer Rohstoffe stellt damit eine wesentliche Voraussetzung für eine funktionierende Volkswirtschaft dar (BMWi 2010: 12).

Kiese und Sande sowie gebrochener Naturstein machen insgesamt rund 75 Prozent aller gewonnenen Rohstoffe in Deutschland aus. Die Abbaumenge beläuft sich auf 465.000 Mio. Tonnen. An dritter Position stehen Kalk-, Mergel- und 
Tabelle 1 Rohstoffproduktion in Deutschland im Jahr 2016 (Angaben in Tonnen; ohne Energierohstoffe und Torf)

\begin{tabular}{ll}
\hline Steine und Erden (gesamt) & $\mathbf{5 3 3 . 6 7 6 . 1 2 9}$ \\
Sand und Kies & 247.000 .000 \\
Gebrochene Natursteine & 218.000 .000 \\
Kalk-, Mergel- und Dolomitstein & 53.193 .000 \\
REA-Gips (Gips, der aus den Abgasen von Rauchgasentschwefelungsanla- & 6.700 .000 \\
gen gewonnen wird) & \\
Lavasand & 5.805 .000 \\
Gips- und Anhydritstein & 3.970 .000 \\
Kreide & 1.700 .000 \\
Bims & 1.200 .000 \\
Eisenerz & 514.000 \\
Naturwerksteine & 430.000 \\
Trass und Tuffstein & 296.000 \\
Dachschiefer & 30.000 \\
Farberde & 129 \\
Industrieminerale (gesamt, darunter Quarzsand und -kies, Sole, Kali- und & $\mathbf{3 8 . 3 9 9 . 8 2 2}$ \\
Kalisalzprodukte) & \\
Metalle (Kupfer, Silber, Gold) & $\mathbf{4 5}$ \\
\hline Quelle: Eigene Berechnungen sowie eigene, veränderte Darstellung auf der Basis von BGR (2017: 18)
\end{tabular}

Dolomitstein mit rund 53.000 Mio. Tonnen (BGR 2017: 15; vgl. Tabelle 1). Viele der in Deutschland benötigten Rohstoffe, vor allem Steine-und-Erden-Rohstoffe, stammen aus heimischen Lagerstätten (Schröder/Vulpius 2013: 4; BGR 2015: 14). Allerdings ist Deutschland in Bezug auf Metallrohstoffe und einige Industriemineralien vom Import abhängig, zudem wurde beispielsweise der Erzabbau im Inland vollständig eingestellt (BGR 2017: 15).

Mineralische Rohstoffe sind standortgebunden und ungleich im Raum verteilt. Sie können erst in bestimmten Konzentrationen und bei entsprechender Größe des Vorkommens mit vertretbarem Aufwand gewonnen werden. Auf der Grundlage von Erkundungen wird die Abbauwürdigkeit von Lagerstätten über mehrere Faktoren nachgewiesen (vor allem Vorratsmenge, Konzentration des Rohstoffes, Erschließungsaufwand, Aufbereitungs- und Transportkosten, Umweltschutzauflagen; Kukuk 1960: 240; Matthes 2001: 7). Ist die Abbauwürdigkeit nicht gegeben, wird von Rohstoffvorkommen gesprochen, deren wirtschaftliche Nutzbarkeit noch nicht hinreichend geklärt werden konnte bzw. gegenwärtig nicht gegeben ist.

Die räumliche Verbreitung der Rohstoffe spiegelt die geologische Entwicklung wider und zeigt deutliche regionale Unterschiede (BGR 2014: 14f.). Bei Kiesen und Sanden, die bundesweit in großen Mengen benötigt werden, gibt es Gebiete mit großen Vorräten, vor allem entlang gröBerer Flüsse, bei eiszeitlichen Ablagerungen in Nord- und Mitteldeutschland sowie im Alpenvorland, aber auch Räume mit Verknappung, z. B. in Teilen der Mittelgebirge und der Norddeutschen Tiefebene. Der Oberrheingraben zwischen Basel und Mainz gilt als größte Kieslagerstätte Europas (LGRB 2013: 27). Die ungleiche Verbreitung führt zur Konzentration von Abbaubereichen und Belastungen, zu weiten Transportwegen und zur Substitution durch andere Rohstoffe.

Es wird geschätzt, dass der Flächenbedarf für die mittel- und langfristige Rohstoffsicherung bei nur etwas über 1 Prozent der Fläche Deutschlands liegt. 2015 wurden nach Angaben des Statistischen Bundesamts rund $1.559 \mathrm{~km}^{2}$ und somit 0,436 Prozent der deutschen Landfläche - als Abbauland genutzt. Die im Jahr 2016 geförderte Rohstoffmenge hat ein Flächenäquivalent von rund $30 \mathrm{~km}^{2}$ (davon 16,3 km² für Baustoffe und Industrieminerale) (BGR 2017: 29). Die Mehrzahl der Abbaustellen weist eine Betriebsgröße von unter 10 ha auf. ${ }^{1}$

Die Bedeutung von Rohstoffen für industrialisierte Gesellschaften ist nicht zu unterschätzen. Dies gilt nicht nur für kritische Rohstoffe, also für solche Rohstoffe, die für die Wirtschaft wichtig sind, aber für die zukünftig ein Versorgungsrisiko bestehen könnte - zumal Rohstoffe höchster oder hoher Kritikalität nicht in Deutschland gewonnen und somit importiert werden (vgl. Erdmann/Behrendt/Feil 2011). Auch die (regionale) Versorgung mit Massenrohstoffen trägt maßgeblich zum Funktionieren von Wirtschaft und Gesellschaft bei. Die Rohstoffinitiative der Europäischen Kommission von 2008 (Kommission der Europäischen Gemeinschaften 2008) wurde im Sinne einer Rohstoffstrategie 2011 (Europäische Kommission 2011) aktualisiert und verfolgt unter anderem das Ziel, eine nachhaltige Rohstoffversorgung aus europäischen Quellen zu unterstützen sowie

\footnotetext{
${ }^{1}$ Schriftliche Befragung des Bundesinstituts für Bau-, Stadt- und Raumforschung (BBSR) zum Stand der Rohstoffsicherung in der Raumordnung (2015/2016).
} 


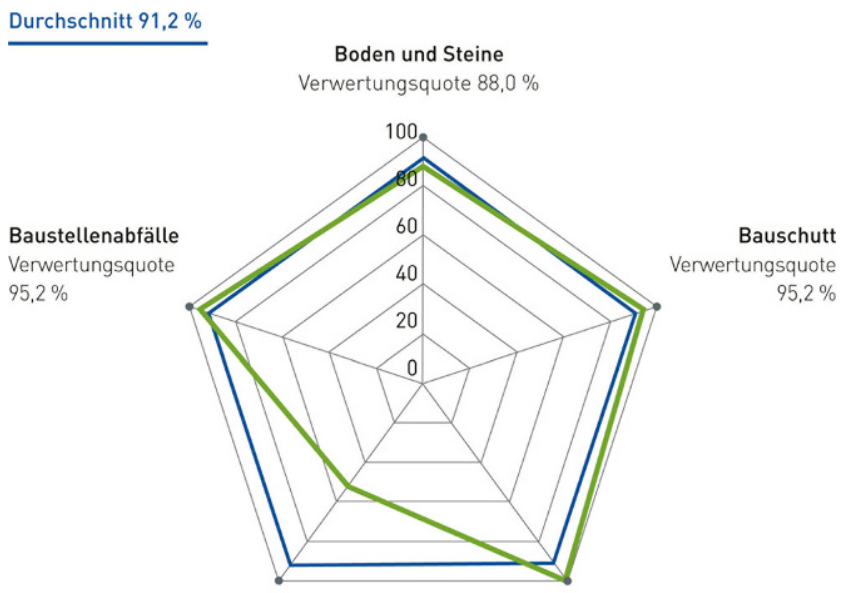

Bauabfälle auf Gipsbasis

Verwertungsquote

$52,1 \%$

Straßenaufbruch

Verwertungsquote

$98,7 \%$

Abbildung 1 Verwertungsquoten mineralischer Bauabfälle 2012 Quelle: Bundesverband Baustoffe - Steine und Erden (2015: 13)

Ressourceneffizienz und Wiederverwertung (Recycling) zu fördern.

Die Rohstoffstrategie der Bundesregierung (BMWi 2010) formuliert vergleichbare Ziele; sie sieht die Sicherstellung der Versorgung mit mineralischen Rohstoffen in Deutschland als eine zentrale öffentliche Aufgabe. Gleichzeitig bleibt es eine Herausforderung, die Wirtschafts- und Produktionsweisen in Deutschland schrittweise von Primärrohstoffen unabhängiger zu machen und die Kreislaufwirtschaft voranzutreiben (BMWi 2010). Deutschland nimmt in der Rückführung von sekundären Rohstoffen aus Abfällen in den Wertstoffkreislauf eine Vorreiterrolle ein. Die Baustoffindustrie setzt diesbezüglich seit Mitte der 1990erJahre Maßstäbe: Bei mineralischen Bauabfällen beträgt die Verwertungsquote rund 90 Prozent (Bundesverband Baustoffe - Steine und Erden 2015: 3; vgl. Abbildung 1). Hohe Recyclingraten werden zudem bei Glas und Papier erzielt, was unter anderem die Gewinnung von Sanden, Kaolin und Kalk betrifft (Bundesverband Baustoffe - Steine und Erden 2016: 36). Für Rohstoffe, die in Deutschland gewonnen werden, sind die Möglichkeiten einer weiteren Steigerung von Substitution und Recycling unter gegenwärtigen Rahmenbedingungen allerdings begrenzt (BMVI 2017: 21f.; vgl. Krenzke 2010; PGRN 2015a; Schröder/Vulpius 2016).

\subsection{Nutzungskonflikte und Flächenkonkurrenzen}

Rohstoffgewinnung ist in aller Regel mit Auswirkungen auf Natur und Landschaft, auf die Flächennutzungen im Umfeld wie auch auf die Bevölkerung verbunden. Konflikte ergeben sich in der Erschließungsphase, während des Abbaus und im Umgang mit Folgenutzungen. Der Rohstoffabbau steht vielerorts in unmittelbarer Flächenkonkurrenz zu anderen Raumnutzungen und Raumfunktionen. Vor al- lem neue Abbauvorhaben stoßen oftmals auf wenig Akzeptanz. Sie können zu heftigem Widerstand der Bevölkerung führen, wenn ein erheblicher Verlust an Landschaftsund Lebensqualität, z. B. durch Nutzung kommunaler StraBen als Transportwege, befürchtet wird, zumal sich der Abbau meist über viele Jahre oder Jahrzehnte erstreckt (vgl. Weber/Jenal/Kühne 2017). In einigen Räumen geht es dabei nicht nur um einzelne Standorte, sondern vielmehr um einen Rohstoffabbau, der durch Vielzahl, Größe oder exponierte Lage seiner Abbaustätten zur Transformation von Landschaften oder Regionen beiträgt. Beispiele hierfür sind der Kiesabbau im Oberrheingraben (LGRB 2013: 35 ff.), der Abbau von Basalten und Lavasanden in der Vulkaneifel (agl 2016: 4 ff.) oder der Kleiabbau in Niedersachsen (Jeschke 2004: 44; Spiekermann 2009: 95). Demgegenüber können sich Abbauvorhaben positiv auf das Arbeitsplatzangebot bzw. die Wertschöpfung in der Region auswirken. Zudem werden ehemalige Abbaustätten, die einer attraktiven Erholungs- oder Freizeitnutzung zugeführt wurden, von der heimischen Bevölkerung und von Touristen häufig gut angenommen und geschätzt.

Umweltrechtliche Restriktionen, Siedlungserweiterungen und gewachsene Flächennutzungskonkurrenzen engen die Spielräume der Rohstoffwirtschaft zunehmend ein. Hinzu kommt, dass schwankende Rohstoff- bzw. Lagerstättenqualitäten, Abbauhindernisse unterschiedlichster Art, wie beispielsweise nach $\S 30 \mathrm{BNatSchG}^{2}$ geschützte Biotope, archäologische Fundstätten oder Hochspannungsmasten, $\mathrm{zu}$ einer Fragmentierung der Potenzialflächen führen und die Rentabilität des Abbaustandorts in Frage stellen können. Die deutschen Rohstoffunternehmen verweisen zudem auf komplizierte und langwierige Genehmigungsverfahren (DERA 2014: 10).

In den letzten Dekaden wurden viele Anstrengungen unternommen, durch Kooperationen und Rahmenvereinbarungen, vorausschauende Planung und verbessertes Standortmanagement die Konfliktsituationen zu entschärfen. So bemühen sich Rohstoffwirtschaft, Naturschutz- und Tourismusverbände sowie Behörden, Synergien und Entwicklungsoptionen während des Abbaus für die Ausgestaltung der Folgenutzungen durch Kooperationsprojekte oder Rahmenvereinbarungen besser zu nutzen.

\section{Rohstoffsicherung als raumordnungspolitisches Handlungsfeld}

Für die Rohstoffgewinnung besteht keine einheitliche Rechtslage in Deutschland. Unter das Bundesberggesetz

\footnotetext{
2 Bundesnaturschutzgesetz vom 29. Juli 2009, zuletzt geändert durch Artikel 1 des Gesetzes vom 15. September 2017.
} 
$(\mathrm{BBergG})^{3}$ fallen in erster Linie bergfreie Bodenschätze, vor allem Erdöl, Erdgas und Kohle, ansonsten greifen andere gesetzliche Regelungen (BGR 2017: 15). Es existiert auch kein eigenes fachplanerisches Instrumentarium zur Rohstoffsicherung, um eine langfristig angelegte Flächenvorsorge $\mathrm{zu}$ betreiben und diese gegenüber anderen Raumnutzungen durchzusetzen. Die Staatlichen Geologischen Dienste Deutschlands sehen deshalb die Belange der Rohstoffsicherung gegenüber anderen, durch formalrechtliche Ausweisungen erhärteten Belangen wie den Natur-, Grundwasser- oder Hochwasserschutz im Nachteil. Sie fordern daher, die Rohstoffsicherung stärker als bislang in den Raumordnungs- und Bauleitplänen zu berücksichtigen (SGD 2008: 24). Diese Forderung wird durch die Rohstoffstrategie der Bundesregierung unterstützt, damit ,bei der Exploration und Gewinnung von Rohstoffen den Interessen der Rohstoffwirtschaft im Rahmen der Raumordnung und Landesplanung sowie bei Genehmigungsverfahren angemessen Rechnung getragen wird" (BMWi 2010: 8).

Gemäß § 2 Abs. 2 ROG $^{4}$ sind im Zuge der Raumordnung ,die räumlichen Voraussetzungen für die vorsorgende Sicherung sowie für die geordnete Aufsuchung und Gewinnung von standortgebundenen Rohstoffen zu schaffen". Gemäß $§ 8$ Abs. 5 S. 2b ROG sollen die Raumordnungspläne Festlegungen zur (Frei-)Raumstruktur enthalten, so unter anderem zu Standorten für die vorsorgende Sicherung sowie die geordnete Aufsuchung und Gewinnung von standortgebundenen Rohstoffen. Hinzu kommt, dass eine aktuelle Änderung raumordnungsrechtlicher Vorschriften die „Raumordnungsklausel“ auch im Bundesberggesetz verankert: In Raumordnungsplänen festgelegte Ziele müssen bei raumbedeutsamen bergrechtlichen Vorhaben beachtet werden, nicht zuletzt um diese raumverträglicher auszugestalten. ${ }^{5}$ Die Raumordnung übernimmt damit eine wichtige Rolle, sowohl in Bezug auf die Rohstoffsicherung und Flächenvorsorge als auch hinsichtlich der Genehmigung neuer Abbauvorhaben.

In den ,Leitbildern und Handlungsstrategien für die Raumentwicklung in Deutschland", beschlossen von der Ministerkonferenz für Raumordnung (MKRO 2016), wird vor allem die Koordinierungsfunktion der Raumordnung betont: „Der Abbau von Rohstoffen soll auch in Zukunft mit konkurrierenden Nutzungsansprüchen, wie etwa der Siedlungsentwicklung oder der Gestaltung von Kulturlandschaften, abgestimmt und auf geeigneten Flächen durch Raumordnungspläne gesichert werden. Zum Schutz der

\footnotetext{
${ }^{3}$ Bundesberggesetz vom 13. August 1980, das durch Artikel 2 Absatz 4 des Gesetzes vom 20. Juli 2017 geändert worden ist.

${ }^{4}$ Raumordnungsgesetz vom 22. Dezember 2008, zuletzt geändert durch Artikel 2 Absatz 15 des Gesetzes vom 20. Juli 2017.

5 Gesetz zur Änderung raumordnungsrechtlicher Vorschriften vom 23. Mai 2017.
}

Naturgüter und für die raumverträgliche Nutzung standortgebundener Bodenschätze bedarf es weiterhin einer vorsorgenden räumlichen und zeitlichen Koordination“ (MKRO 2016: 26).

\section{Stand der Rohstoffsicherung in der Raumordnung}

Das Bundesinstitut für Bau-, Stadt- und Raumforschung (BBSR) führte 2015/2016 eine schriftliche Befragung zum Stand der Rohstoffsicherung in der Raumordnung durch. Befragt wurden die Träger der Landes- und Regionalplanung, der Geologischen Dienste der Länder sowie Akteure der Rohstoffwirtschaft. Die Befragung der Rohstoffwirtschaft erfolgte 2016 zentral über den Bundesverband Baustoffe - Steine und Erden e.V. (bbs), die Vereinigung Rohstoffe und Bergbau e.V. (VRB) sowie den Verband der Kaliund Salzindustrie e.V. (VKS) (vgl. BMVI 2017).

Im Rahmen des MORO-Vorhabens ,Mittel- und langfristige Sicherung mineralischer Rohstoffe in der landesweiten Raumplanung und in der Regionalplanung“ (BMVI 2017) wurde zudem eine Auswertung der Raumordnungspläne der Länder vorgenommen; Ziel war, insbesondere die Vorgaben für die Regionalplanung nachvollziehen zu können. Um spezifische Aspekte der Rohstoffsicherung auf regionaler Ebene näher zu beleuchten, erfolgte eine Analyse von 15 Regionalplänen. Diese wurden unter anderem aufgrund spezifischer methodischer Ansätze in der Abwägung (z. B. Ermittlung der Eignung der Rohstoffflächen, Bewertung der Raumverträglichkeit, Einschätzung der Wirkung räumlicher Konzentrationen von Abbauflächen, Bestimmung von Mengenzielen) sowie eines breiten Spektrums an raumordnerischen Instrumenten oder weitgehender Regelungen für Folgenutzungen ausgewählt.

Sowohl die Befragungen als auch die Plananalysen haben bestätigt, dass die raumordnerische Rohstoffsicherung auf Landes- und auf regionaler Ebene sehr unterschiedlich gehandhabt wird (BMVI 2017: vgl. auch SGD 2008: 11; Schröder/Vulpius 2016: 67). Bereits die fachlichen Informationsgrundlagen variieren demnach in erheblichem Umfang. Zwar verwenden die Geologischen Dienste der Länder vergleichbare Kriterien zur Bewertung von Lagerstätten, allerdings sind die Grundlagendaten in Bezug auf die Abbauwürdigkeit sowie die Qualität und Mächtigkeit der Rohstoffe auf einem sehr unterschiedlichen Stand. Gerade in den östlichen Bundesländern können die Geologischen Dienste auf eigene Erkundungen noch aus den Zeiten vor der Wiedervereinigung zurückgreifen, wohingegen in den westlichen Bundesländern meist die Daten der Unternehmen, sofern zugänglich, herangezogen werden müssen (vgl. auch SGD 2008). Die Geologischen Dienste der Länder schätzen Umfang und Qualität der vorhandenen Informati- 
onsgrundlagen, vor allem hinsichtlich der langfristigen Sicherung von Rohstoffpotenzialen, deutlich kritischer als die Planungsträger ein. Gleichwohl sehen auch die Planungsträger Informationslücken in Bezug auf Abbaumengen, Größe der Abbauflächen sowie Ausschöpfungsgrad der Vorkommen. Dies betrifft das Monitoring des Abbaus, da es für die nicht unter Bergaufsicht stehenden Flächen und für Betriebe unter einer bestimmten Mindestgröße meist keinen gesicherten und zentral vorgehaltenen Datenpool gibt.

Das Ergebnis der Befragung zur Bewertung des aktuellen Stands der Rohstoffsicherung in der Raumordnung vermittelt in vielen Bereichen ein insgesamt heterogenes, lückenhaftes Gesamtbild, das durch die Plananalyse erhärtet wird. Ermittelte Verbesserungsvorschläge betreffen unter anderem die Qualität der Informationsgrundlagen, die inhaltliche Abwägung, die Sicherungszeiträume oder die Beteiligung weiterer Akteure. Vorschläge zu raumordnerischen Instrumenten zielen insbesondere auf eine Verbesserung der langfristigen Sicherung ab.

Die Rohstoffwirtschaft gibt zu bedenken, dass die vergleichsweise kurzen Geltungszeiträume der Raumordnungspläne nicht mit den oftmals langfristigen Planungshorizonten der Abbauvorhaben korrespondieren, dass die Festlegungen, sofern es sich nicht um Vorranggebiete handelt, den Unternehmen keine ausreichende Planungssicherheit bieten und dass die Möglichkeit einer bedarfsunabhängigen, vorsorgenden Sicherung der Rohstoffvorkommen eingeräumt werden sollte (Bundesverband Baustoffe Steine und Erden 2010: 20 f.: Schröder/Vulpius 2016: 68; Schröder 2017: 66). In der Befragung der Rohstoffverbände (2016) wurden diese Aspekte nochmals betont. Zudem sehen die Rohstoffverbände insbesondere die Landesplanung als wichtigen Akteur (vgl. BMVI 2017: 49). Die Landesplanung sollte beide raumordnerischen Teilaufgaben wahrnehmen, das heißt sowohl die Voraussetzungen für die Aufsuchung und Gewinnung von Rohstoffen schaffen als auch der vorsorgenden Rohstoffsicherung nachkommen, und möglichst konkrete Vorgaben für die regionale Ebene formulieren. Die Flächenvorsorge sollte dabei in erster Linie über die Festlegung von Vorranggebieten erfolgen (vgl. auch Schröder 2017: 66). Die Ausgestaltung der Instrumentarien zur Rohstoffsicherung auf Landes- und regionaler Ebene wird je nach Bundesland sehr unterschiedlich bewertet (vgl. auch Schröder/Vulpius 2016).

Mindestsicherungsmengen oder -ziele werden von den Rohstoffverbänden kritisch bewertet. Sie sollten lediglich als Hilfestellung zur methodischen Abgrenzung der Rohstoffgewinnungsgebiete herangezogen werden. In Bezug auf die Datengrundlagen sollte das Niveau anderer Fachplanungen erreicht werden, um in der Abwägung gegenüber anderen Belangen ein angemessenes Gewicht zu entfalten (vgl. BMVI 2017: 52). Um die Planungssicherheit für die Unternehmen zu erhöhen, wird eine Verlängerung der raumplanerischen Flächensicherung als erforderlich angesehen: Für die Rohstoffgewinnung sollten mindestens 25 Jahre veranschlagt (Schröder 2017: 67), für die vorsorgende Rohstoffsicherung zeitlich unbefristete und bedarfsunabhängige Festlegungen getroffen werden.

Das Landesentwicklungsprogramm Thüringen greift diesen Ansatz in den Vorgaben für die Träger der Regionalplanung auf (TMBLV 2014: 12, Begründung zu V 6.3.5 und 6.3.6): „Damit eine bedarfsgerechte und möglichst verbrauchernahe Rohstoffversorgung [...] gewährleistet werden kann, ist eine diesem Anliegen angemessene Flächensicherung durch Vorrang- und Vorbehaltsgebiete, Rohstoffgewinnung' erforderlich.... Die für eine wirtschaftliche Gewinnung der Rohstoffe erforderlichen Investitionen und laufenden Ersatzinvestitionen erfordern in der Regel eine Laufzeit von mindestens 25 Jahren. Dies gilt es, insbesondere bei der Bemessung der Größe der Vorranggebiete ,Rohstoffgewinnung', zu berücksichtigen." Die vorsorgende Rohstoffsicherung zielt hingegen auf eine Sicherung vorhandener Rohstoffpotenziale für künftige Generationen und soll bedarfsunabhängig erfolgen.

Die Ergebnisse der Plananalyse bilden die Bandbreite raumordnerischer Festlegungen ab. In einem Großteil der untersuchten Landesraumordnungspläne werden zwar beide Teilaufgaben, die Rohstoffgewinnung wie auch die langfristige Rohstoffsicherung, angesprochen, allerdings füllen die Länder diese Aufgaben sehr unterschiedlich aus. Das Spektrum reicht von wenigen rahmensetzenden Vorgaben bzw. Grundsätzen bis zu weitreichenden und letztabgewogenen Zielen (vgl. auch Schröder/Vulpius 2016: 68; Schröder 2017: 63). Teilweise werden raumordnerische Zielfestlegungen räumlich konkretisiert, beispielsweise als „Vorranggebiete Rohstoffgewinnung“ oder „Vorranggebiete Rohstoffsicherung“. Wird bereits auf Länderebene konkret ausgewiesen, welche Rohstoffvorkommen in der Regionalplanung gesichert werden sollen bzw. welche Instrumente zur Rohstoffsicherung anzuwenden sind, trägt dies zu einem einheitlichen Vorgehen auf regionaler Ebene bei. Gleichzeitig werden damit die Spielräume der Regionalplanung für eine an die regionalen Rahmenbedingungen angepasste räumliche und zeitliche Steuerung der Rohstoffsicherung erheblich eingeengt.

Viele Landesraumordnungspläne formulieren Vorgaben, um auf eine möglichst konfliktarme Standortwahl, eine umweltgerechte Nutzung mit vollständiger Ausbeutung der Abbaustätten, eine zeitnahe Rekultivierung oder konkrete Folgenutzungen hinzuwirken. Zwischennutzungen, wie beispielsweise die Gewinnung erneuerbarer Energien, thematisieren bislang nur wenige Bundesländer. Die Regionalpläne greifen darüber hinaus in der Regel die oftmals bereits auf der Landesebene formulierten Vorgaben für eine raumverträgliche Rohstoffsicherung auf und versuchen, Synergien zwischen Rohstoffsicherung und Naturschutz, Landschafts- 
Abbildung 2 Auf welchen Flächen wird die Ausweisung von Vorranggebieten für die Rohstoffsicherung ausgeschlossen? Quelle: Eigene Darstellung auf der Basis von BMVI (2017: 39)

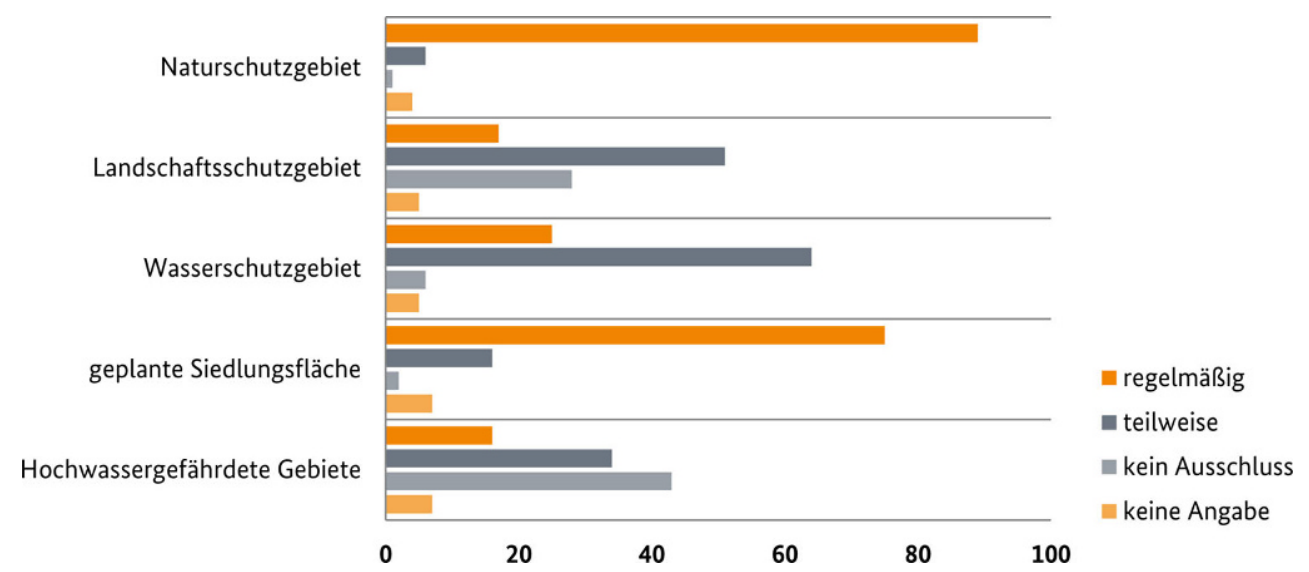

schutz, Hochwasservorsorge oder auch anderen Landnutzungen wie der Landwirtschaft zu erzielen.

Die Regionalplanung sichert Rohstoffpotenziale in erster Linie über Vorranggebiete für die Rohstoffgewinnung sowie Vorbehaltsgebiete für die langfristige Sicherung, wobei die Plansätze und räumlichen Festlegungen sehr unterschiedlich ausfallen (vgl. von Seht 2010: $421 \mathrm{ff}$.). In einzelnen Bundesländern werden auf regionaler Ebene spezifische Instrumente eingesetzt, z. B. Vorranggebiete mit der Wirkung von Eignungsgebieten, Ausschlussgebiete, Reservegebiete oder Vorranggebiete zur langfristigen Sicherung. Die Vorranggebiete für die Rohstoffsicherung werden teilweise mit einem Ausschluss für die Rohstoffgewinnung kombiniert.

Bei der raumordnerischen Standortfindung werden verschiedene methodische Ansätze verfolgt. In der Regel erfolgt der Ausschluss von Flächen für die Rohstoffgewinnung bzw. Rohstoffsicherung über konkurrierende fachplanerische Schutzkategorien bzw. bauleitplanerische Festlegungen (vgl. Abbildung 2). Auch Nachfrageprognosen und Potenzialanalysen spielen eine wesentliche Rolle. Ein systematisches Verfahren zur Bewertung der Eignung von Rohstoffpotenzialflächen, zur Prüfung der Raumverträglichkeit sowie zur Priorisierung der Flächenkontingente auf regionaler Ebene erhöhen die Transparenz des Abwägungsverfahrens und die Belastbarkeit der Ergebnisse.

Eine Qualifizierung des Abwägungsprozesses kann durch die intensive Mitwirkung regionaler Akteure und Fachbehörden erzielt werden. Eine enge fachplanerische Abstimmung ist vor allem für die Bewertung der Rohstoffvorkommen und die Ermittlung der Flächenerfordernisse für die Rohstoffgewinnung erforderlich. Darüber hinaus kann es zielführend sein, eine Abstimmung auf informellem Wege bereits im Vorfeld der formalen Planung herbeizuführen.

\section{Konzeptionelle Überlegungen zur Rohstoffsicherung in der Raumordnung}

Konzeptionelle Ansätze zur zukünftigen Ausgestaltung der Rohstoffsicherung in der Raumordnung werden vor allem im Hinblick auf die Grundlagendaten, das Verfahren der raumordnerischen Abwägung und die Ausgestaltung der raumordnerischen Instrumente zur Rohstoffsicherung gesehen. Die folgenden konzeptionellen Überlegungen werden durch Beispiele ergänzt, sofern diese bereits in der Praxis zur Anwendung kommen.

\subsection{Anforderungen an die Grundlagendaten und deren Aufbereitung}

\subsubsection{Rohstoffgeologische Informationen}

Die für die Planungsträger erforderlichen rohstoffgeologischen Informationen sollten nach den Ergebnissen der Befragungen (vgl. BMVI 2017) folgende Datensätze umfassen:

- eine valide und nachvollziehbare fachliche Abgrenzung und Bewertung der Rohstoffpotenzialflächen (Rohstoffvorkommen/-lagerstätten),

- eine aktuelle Erhebung der genehmigten Abbauflächen und der verbleibenden Rohstoffmengen,

- eine Bestimmung des Bedarfs für die Rohstoffgewinnung im Planungszeitraum zur methodischen Abgrenzung gegenüber einer langfristigen (bedarfsunabhängigen) Rohstoffsicherung.

Hier besteht deutlicher Optimierungsbedarf, da die Datenlage zur Rohstoffsicherung im Vergleich zu anderen Sektorpolitiken, wie dem Naturschutz oder der Wasserwirtschaft, oftmals deutliche Defizite aufweist bzw. Daten nicht zur Verfügung stehen (vgl. auch Krumm 2014: 6ff.). Hier sind beispielsweise fehlende bzw. unzureichende Informationen über den genehmigten Rohstoffabbau (Um- 


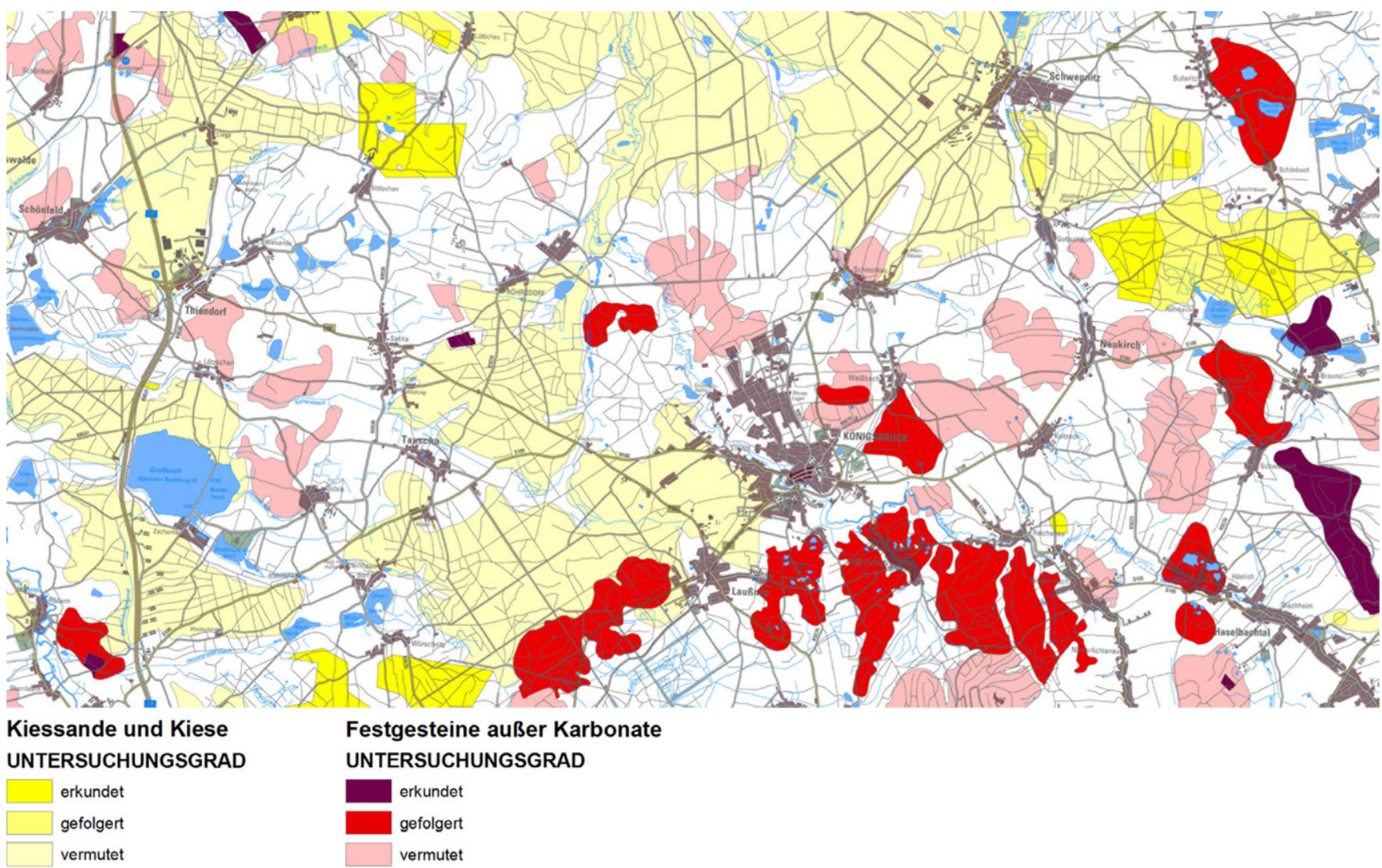

Abbildung 3 Ausschnitt aus der Karte der oberflächennahen mineralischen Rohstoffe 1:50.000 (KOR 50) von Sachsen (Ausschnitt nicht maßstabsgetreu) Quelle: Sächsisches Landesamt für Umwelt, Landwirtschaft und Geologie (LfULG), Referat Rohstoffgeologie

griff, Mengen), zu noch vorhandenen Restmengen in den genehmigten Abbauflächen oder zur Eignung der Rohstoffpotenzialflächen zu nennen.

Die Bundesanstalt für Geowissenschaften und Rohstoffe fordert, dass „das geologisch-lagerstättenkundliche Wissen über die oberflächennahen Rohstoffvorkommen [...] durch verstärkte Prospektions- und Explorationsarbeiten zukünftig weiter aktualisiert und vertieft werden [muss], um gegenüber anderen raumbeanspruchenden Nutzungen mit gleichermaßen detaillierten und belastbaren Sachinformationen aufwarten zu können" (BGR 2015: 25). Die EU-Kommission empfiehlt deshalb, „die staatlichen geologischen Anstalten stärker an der Raumplanung der Mitgliedstaaten zu beteiligen“ (Kommission der Europäischen Gemeinschaften 2008: 10). In einigen Bundesländern gibt es bereits eine systematische und flächendeckende Bestimmung und Darstellung von Eignung bzw. Bauwürdigkeit der Rohstoffe. So hält das digitale „Fachinformationssystem Rohstoffe" (FIS-Ro) in Sachsen zahlreiche Informationen unter anderem zu Rohstoffarten, Bauwürdigkeit, Betriebs- stellen, Rechtsverhältnissen und Wiedernutzbarmachung vor (vgl. Abbildung 3). ${ }^{6}$

Für bereits genehmigte Abbauflächen erfolgt in den Raumordnungsplänen in aller Regel eine nachrichtliche Übernahme bzw. eine Integration in die Vorranggebiete zur Rohstoffgewinnung. Die Informationen zu Abbauflächen, Förder- und verbleibenden Rohstoffmengen sowie zu Rekultivierungen sind aufgrund der Genehmigung nach unterschiedlichen Fachgesetzen meist unvollständig und uneinheitlich. Vor allem in konfliktreichen Verfahren erschwert eine unvollständige Informationsgrundlage den Verhandlungs- und Abwägungsprozess, was zu einem Vertrauensverlust in die Planung insgesamt führen bzw. die Rechtssicherheit in Frage stellen kann. Eine Mitteilungspflicht zu genehmigten Abbauflächen und ihrem Abbaustatus könnte hier Abhilfe schaffen. Dazu sollte ein einheitliches Monitoring von Seiten der staatlichen Behörden gewährleistet werden (vgl. SGD 2008: 24). Ein Beispiel sind die Maßnahmen des Geologischen Dienstes

$6 \mathrm{Vgl}$. www.umwelt.sachsen.de/umwelt/geologie/8092.htm 
Abbildung 4 Zuschläge zur Festlegung von Sicherungsgebieten für den Rohstoffabbau in der Region Rheinhessen-Nahe Quelle: Eigene Darstellung auf der Basis von PGRN (2015a: 18)

\begin{tabular}{|c|c|c|c|c|c|c|c|}
\hline $\begin{array}{l}\text { Abbau- } \\
\text { menge } \\
(1.000 t)\end{array}$ & Rohstoffart & Jahre & $\begin{array}{l}15 \text { Jahre } \\
\text { Planungs- } \\
\text { horizont } \\
(1.000 t)\end{array}$ & $\begin{array}{l}\text { Zuschlag } 1 \\
\text { Genehmigungs- } \\
\text { unsicherheiten } \\
\text { Flächenverfüg- } \\
\text { barkeit }\end{array}$ & $\begin{array}{l}\text { Zuschlag } 2 \\
\text { Minderqualitäten/ } \\
\text { Nutzschicht-Ab- } \\
\text { raumverhältnis }\end{array}$ & $\begin{array}{l}\text { Summe } \\
\text { Zuschläge }\end{array}$ & $\begin{array}{l}\text { Mengen- } \\
\text { zielwert } \\
(1.000 \mathrm{t})\end{array}$ \\
\hline $1.605,0$ & Andesit & 15 & $24.075,0$ & $20 \%$ & $30 \%$ & $50 \%$ & $36.112,5$ \\
\hline 5,5 & $\begin{array}{l}\text { Dach- } \\
\text { schiefer }\end{array}$ & 15 & 82,5 & $20 \%$ & $30 \%$ & $50 \%$ & 123,75 \\
\hline 450,0 & Kalkstein & 15 & $6.750,0$ & $20 \%$ & $30 \%$ & $50 \%$ & $10.125,0$ \\
\hline $1.816,3$ & $\begin{array}{l}\text { Kies und } \\
\text { Sand }\end{array}$ & 15 & $27.244,3$ & $20 \%$ & $30 \%$ & $50 \%$ & $40.866,5$ \\
\hline $1.342,0$ & Quarzit & 15 & $20.130,0$ & $20 \%$ & $30 \%$ & $50 \%$ & $30.195,0$ \\
\hline 157,5 & Quarzsand & 15 & $2.361,9$ & $20 \%$ & $30 \%$ & $50 \%$ & $3.542,8$ \\
\hline 107,4 & $\begin{array}{l}\text { Quarzsand } \\
\text { und Kies }\end{array}$ & 15 & $1.611,0$ & $20 \%$ & $30 \%$ & $50 \%$ & $2.416,5$ \\
\hline 934,2 & Rhyolith & 15 & $14.012,6$ & $20 \%$ & $30 \%$ & $50 \%$ & $21.018,0$ \\
\hline 0 & Sandstein & 15 & k.A. & $20 \%$ & $30 \%$ & $50 \%$ & k.A. \\
\hline 95,4 & $\begin{array}{c}\text { Ton-I } \\
\text { Schluffstein }\end{array}$ & 15 & $1.431,7$ & $20 \%$ & $30 \%$ & $50 \%$ & $2.147,5$ \\
\hline $\begin{array}{r}\text { unbekannt } \\
\text { (wenig) }\end{array}$ & Löss & 15 & k.A. & $20 \%$ & $30 \%$ & $50 \%$ & k.A. \\
\hline
\end{tabular}

Die nach Rohstoffarten differenzierte Bedarfsermittlung kann auf der Grundlage tatsächlicher Fördermengen und darauf beruhenden Trendfortschreibungen erfolgen. Sie kann auch auf Basis einer prognostizierten (gesamt-)wirtschaftlichen Entwicklung des Rohstoffbedarfs im Rahmen von Modellrechnungen (,angebotsorientiert ${ }^{\circ}$ ) vorgenommen werden. Die ermittelten Rohstoffmengen korrespondieren entsprechend dem spezifischen Gewicht der Rohstoffart $\left(\mathrm{t} / \mathrm{m}^{3}\right)$ und der geschätzten Mächtigkeit des Rohstoffvorkommens bzw. der möglichen Abbauhöhe mit Fördervolumina. Über diese wiederum errechnet sich die zu sichernde Fläche, möglichst unter Berücksichtigung des NutzschichtAbraumverhältnisses sowie der Abbaugeometrien. Die Berechnungen sind mit erheblichen Unsicherheiten behaftet, weshalb in der Regionalplanung meist Zuschläge zum Einsatz kommen, die Genehmigungsunsicherheiten, mangelnde Flächenverfügbarkeiten, mögliche Minderqualitäten oder ein ungünstiges Nutzschicht-Abraumverhältnis ausgleichen sollen (vgl. Abbildung 4). Für die Sicherheitszuschläge können je nach Rohstoffart bis zu 200 Prozent veranschlagt werden.

Vor diesem Hintergrund erscheinen eine bundesweit einheitliche Vorgehensweise bzw. Mindeststandards in Bezug auf die Erkundung und Erhebung, die fachliche Bewertung der Rohstoffpotenzialflächen, die rohstoffartendifferenzierte Bedarfsermittlung sowie das Monitoring der Rohstoffgewinnung für die Raumordnung sinnvoll. Die Einhaltung von Mindeststandards könnte dazu beitragen, die Rohstoffsicherung besser in den Verfahren der Raumordnung zu verankern.

7 Vgl. http://www.gd.nrw.de/ro_am.htm (15.03.2018).

${ }^{8}$ Raumordnungsverordnung vom 13. Dezember 1990, die zuletzt durch Artikel 5 Absatz 35 des Gesetzes vom 24. Februar 2012 geändert worden ist. 
Tabelle 2 Operationalisierung von Bedeutung und Empfindlichkeit der Schutzgüter zur Bestimmung des Raumwiderstands

\section{Raumwiderstandskriterien der Kategorie} I (,Ausschlusskriterien“)

Hierunter fallen Flächen, deren geplante oder aktuelle Widmung (Nutzung, Funktion) aufgrund überwiegend fachgesetzlicher Regelungen mit einem potenziellen Rohstoffabbau nicht vereinbar und einer Abwägung durch die Raumordnung nicht zugänglich ist. Sie kennzeichnen „Tabuflächen“ und führen zum Ausschluss der Rohstoffpotenzialfläche aus dem für die Raumordnung relevanten Flächenkontingent oder zumindest zum Ausschluss der betroffenen Teilbereiche.

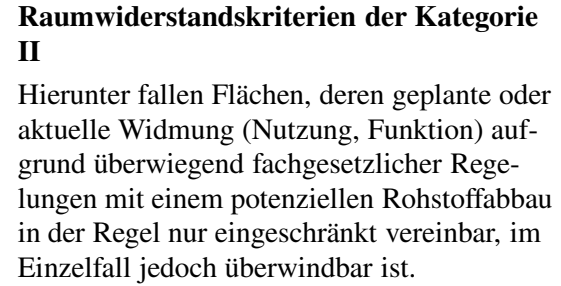

Hierunter fallen Flächen, deren geplante oder aktuelle Widmung (Nutzung, Funktion) aufgrund überwiegend fachgesetzlicher Regelungen mit einem potenziellen Rohstoffabbau in der Regel nur eingeschränkt vereinbar, im Einzelfall jedoch überwindbar ist.

Raumwiderstandskriterien der Kategorie III

Hierunter fallen Flächen, deren geplante oder aktuelle Widmung (Nutzung, Funktion) aufgrund überwiegend fachgesetzlicher Regelungen einem potenziellen Rohstoffabbau in der Regel nicht grundsätzlich entgegensteht, jedoch Berücksichtigung finden muss.

Quelle: Eigene Darstellung auf der Basis von BMVI (2017: 87)

\subsubsection{Daten zur Bestimmung des Raumwiderstands}

Das Gesetz über die Umweltverträglichkeitsprüfung $(\mathrm{UVPG})^{9}$ setzt in $\S 2$ Abs. 1. S. 2 fest, dass Auswirkungen von Vorhaben auf bestimmte Schutzgüter im Rahmen einer Umweltverträglichkeitsprüfung ermittelt, beschrieben und bewertet werden müssen. Auch für die vorsorgende Planung stehen diese Schutzgüter im Vordergrund der Betrachtung. Hierzu zählen gemäß $§ 2$ Abs. 1 UVPG der Mensch bzw. die menschliche Gesundheit, Tiere, Pflanzen und biologische Vielfalt, Boden, Wasser, Luft, Klima und Landschaft, Kultur- und sonstige Sachgüter sowie Wechselwirkungen zwischen den Schutzgütern. In Abhängigkeit von der Empfindlichkeit oder Schutzwürdigkeit bzw. Bedeutung der Schutzgüter lässt sich der „Raumwiderstand“ gegenüber spezifischen Vorhaben oder Eingriffen wie dem Rohstoffabbau bestimmen.

Um den Abwägungsprozess transparent und belastbar zu gestalten, bietet sich auf der Ebene der Regionalplanung eine Operationalisierung von Bedeutung und Empfindlichkeit der Schutzgüter über ein Set an Indikatoren oder Kriterien an. Damit lässt sich der Raumwiderstand gegenüber spezifischen Vorhaben, hier der Rohstoffgewinnung bzw. -sicherung, darstellen. Als methodischer Ansatz wird vorgeschlagen, eine Einteilung in mindestens drei Kategorien vorzusehen (vgl. Tabelle 2; PGRN 2015a; BMVI 2017). Zur Ermittlung des Raumwiderstands bietet sich eine Addition zu einem Konfliktwert pro Fläche an, wobei eine Gewichtung der Kriterien der Kategorien II und III vorgenommen werden sollte, um der unterschiedlichen Konfliktschwere Rechnung zu tragen. Der so ermittelte Konfliktwert beschreibt den Raumwiderstand der betreffenden Fläche. Darüber hinaus können pauschalierte Abstände (Puffer) für sensitive Nutzungen wie öffentliche Einrichtungen und Wohngebiete sowie kritische Infrastrukturen angesetzt

\footnotetext{
${ }^{9}$ Gesetz über die Umweltverträglichkeitsprüfung in der Fassung der Bekanntmachung vom 24. Februar 2010, das zuletzt durch Artikel 2 des Gesetzes vom 8. September 2017 geändert worden ist.
}

werden, um potenzielle Auswirkungen des Rohstoffabbaus, wie beispielsweise Lärm- oder Staubemissionen, in die Abwägung einzubeziehen.

Die Grundlagendaten zu Schutzgütern, sensitiven Nutzungen oder kritischen Infrastrukturen werden in der Regel von den zuständigen Fachplanungen zur Verfügung gestellt. Die Ausgestaltung der Kriterien und deren potenzielle Gewichtung sowie die Integration pauschalierter Abstände müssen an die spezifischen regionalen Kontexte angepasst werden. Eine sektorübergreifende Abstimmung kann insbesondere bei konfliktreichen Situationen die Belastbarkeit der Ergebnisse deutlich verbessern. Ein Beispiel gibt das Pilotvorhaben der Planungsgemeinschaft Rheinhessen-Nahe: Hier wurden sogenannte Raumwiderstandskriterien im Rahmen von Akteurworkshops mit den betroffenen Fachplanungen abgestimmt (PGRN 2015a: 24; PGRN 2015b: 75 ff.; vgl. Abbildung 5).

Die Bedeutung der fachplanerischen Grundlagendaten und Bewertungen zeigt sich am Beispiel der Natura 2000Flächenkulisse (FFH-Gebiete und EU-Vogelschutzgebiete). Bei den Flächen ist zwar nicht a priori von einer Unvereinbarkeit mit dem Rohstoffabbau auszugehen, allerdings schreiben Art. 6 Abs. 3 FFH-RL ${ }^{10}$ bzw. § 34 BNatSchG eine naturschutzfachliche Prüfung der Verträglichkeit von Projekten oder Plänen vor, die die festgelegten Erhaltungsziele erheblich beeinträchtigen können. Sollte eine Vereinbarkeit des Vorhabens mit den festgelegten Erhaltungszielen des betreffenden Gebiets nicht gegeben sein, ist diese fachplanerische Bewertung einer raumordnerischen Abwägung nicht zugänglich. Insofern können nur dann überlagernde Vorranggebiete für die Rohstoffsicherung rechtssicher festgelegt werden, wenn eine fachplanerische Prüfung eine prinzipielle Vereinbarkeit der Erhaltungsziele mit einem potenziellen Rohstoffabbau feststellt (vgl. Düppenbecker/Greiving 1999).

\footnotetext{
${ }_{10}$ Richtlinie 92/43/EWG des Rates vom 21. Mai 1992 zur Erhaltung der natürlichen Lebensräume sowie der wildlebenden Tiere und Pflanzen.
} 


\begin{tabular}{|c|c|c|c|}
\hline $\begin{array}{l}\text { RWK I } \\
\text { (Bestand und Planung/ } \\
\text { im Ausweisungsverfahren) }\end{array}$ & $\begin{array}{l}\text { RWK la } \\
\text { (Bestand und Planung/ } \\
\text { im Ausweisungsverfahren) }\end{array}$ & $\begin{array}{l}\text { RWK II } \\
\text { (Bestand und Planung/ } \\
\text { im Ausweisungsverfahren) } \\
\text { Gewichtungsfaktor } 2\end{array}$ & $\begin{array}{l}\text { RWK III } \\
\text { (Bestand und Planung/ } \\
\text { im Ausweisungsverfahren) } \\
\text { Gewichtungsfaktor } 1\end{array}$ \\
\hline \multicolumn{4}{|l|}{ Wasser } \\
\hline $\begin{array}{l}\text { - WSG Zone I und II } \\
\text { - Heilquellenschutzgebiet } \\
\text { (abhängig von Festset- } \\
\text { zungen in der Verord- } \\
\text { nung) } \\
\text { - Gewässer 1. und } 2 \text {. } \\
\text { Ordnung } \\
\text { - Hochwasserschutz- } \\
\text { damm }\end{array}$ & $\begin{array}{l}\text { - WSG Zone IIla } \\
\text { (sofern keine Untertei- } \\
\text { lung in IIIa/b } \rightarrow \text { gesamte } \\
\text { Zone III als RWK la) }\end{array}$ & $\begin{array}{l}\text { - WSG Zone IIlb } \\
\text { - Landesweit bedeutsa- } \\
\text { me Ressourcen für den } \\
\text { Grundwasserschutz und } \\
\text { die Trinkwassergewin- } \\
\text { nung - Bereiche von her- } \\
\text { ausragender Bedeutung } \\
\text { - Gewässer 3. Ordnung } \\
\text { - Heilquellenschutzgebiet } \\
\text { (abhängig von Festsetzun- } \\
\text { gen in der Verordnung) }\end{array}$ & $\begin{array}{l}\text { - Landesweit bedeutsa- } \\
\text { me Ressourcen für den } \\
\text { Grundwasserschutz und } \\
\text { die Trinkwassergewin- } \\
\text { nung - Bereiche von } \\
\text { besonderer Bedeutung } \\
\text { - Gesetzlich festgesetzte } \\
\text { Überschwemmungsge- } \\
\text { biete }\end{array}$ \\
\hline \multicolumn{4}{|l|}{ Boden } \\
\hline $\begin{array}{l}\text { - Bodenbelastungs- und } \\
\text { Bodenschutzgebiete } \\
\text { gemäß § } 8 \text { LBodSchG² }\end{array}$ & & $\begin{array}{l}\text { - Bodenbelastungs- und } \\
\text { Bodenschutzgebiete } \\
\text { gemäß § } 8 \text { LBodSchG² }\end{array}$ & $\begin{array}{l}\text { - Böden mit hoher und } \\
\text { sehr hoher natürlicher } \\
\text { Bodenfruchtbarkeit } \\
\text { - Seltene Böden bzw. Bö- } \\
\text { den als Archive der Natur- } \\
\text { und Kulturgeschichte }{ }^{3}\end{array}$ \\
\hline \multicolumn{4}{|c|}{ Fauna, Flora, biologische Vielfalt } \\
\hline $\begin{array}{l}\text { - NSG } \\
\text { - FFH-/ Vogelschutzgebiet } \\
\text { und Umgebung, laut } \\
\text { Voreinschätzung erhebli- } \\
\text { che Beeinträchtigung der } \\
\text { Erhaltungsziele sicher } \\
\text { - Nationalpark } \\
\text { - § 30-Biotope hoher } \\
\text { Dichte und mit hohem } \\
\text { Flächenanteil, zentrale/ } \\
\text { disperse Lage, herausra- } \\
\text { gende Qualität }{ }^{5}\end{array}$ & $\begin{array}{l}\text { - FFH-/ Vogelschutzgebiet } \\
\text { und Umgebung sofern } \\
\text { keine Voreinschätzung } \\
\text { erfolgt }^{4}\end{array}$ & $\begin{array}{l}\text { - FFH-/ Vogelschutzgebiet } \\
\text { und Umgebung, laut } \\
\text { Voreinschätzung Zuläs- } \\
\text { sigkeit möglich } \\
\text { - Sehr bedeutende Flächen } \\
\text { des regionalen Biotop- } \\
\text { verbundes } \\
\text { - § 30-Biotope mittlerer } \\
\text { Dichte und mit mittlerem } \\
\text { Flächenanteil, randliche }_{\text {Lage }^{5}}\end{array}$ & $\begin{array}{l}\text { - FFH-/ Vogelschutzgebiet } \\
\text { und Umgebung, laut } \\
\text { Voreinschätzung keine } \\
\text { negativen Auswirkungen } \\
\text { auf Erhaltungsziele } \\
\text { - Bedeutende Flächen des } \\
\text { regionalen Biotopver- } \\
\text { bundes } \\
\text { - § } 30-\text { Biotope geringer } \\
\text { Dichte und mit geringem } \\
\text { Flächenanteil, randliche } \\
\text { Lage }^{5}\end{array}$ \\
\hline \multicolumn{4}{|l|}{ Landschaft } \\
\hline $\begin{array}{l}\text { - Limes (Kern- und Rah- } \\
\text { menzone) } \\
\text { - Kernzone Naturpark } \\
\text { - Kernzone Biosphärenre- } \\
\text { servat }\end{array}$ & & $\begin{array}{l}\text { - Kernzone UNESCO } \\
\text { Welterbe } \\
\text { - Naturpark außerhalb } \\
\text { Kernzone } \\
\text { - Pflegezone Biosphären- } \\
\text { reservat, Ausnahme bei } \\
\text { Pfälzerwald Pflege- und } \\
\text { Entwicklungszone } \\
\text { - Landesweit bedeutsame } \\
\text { Kulturlandschaften mit } \\
\text { herausragender und sehr } \\
\text { hoher/ hoher Bedeutung } \\
\text { - Hohe Dichte und hoher } \\
\text { Flächenanteil, zentrale/ } \\
\text { disperse Lage von ge- } \\
\text { schützten Landschaftsbe- } \\
\text { standteilen/ Naturdenk- } \\
\text { malen }\end{array}$ & $\begin{array}{l}\text { - Entwicklungszone Bio- } \\
\text { sphärenreservat } \\
\text { - Landesweit bedeutsame } \\
\text { Kulturlandschaften mit } \\
\text { gehobener Bedeutung } \\
\text { - Regional repräsenta- } \\
\text { tive und bedeutsame } \\
\text { Landschaftsbestandteile } \\
\text { (LRPL) } \\
\text { - Geringe Dichte und } \\
\text { geringer Flächenanteil, } \\
\text { randliche Lage von } \\
\text { geschützten Landschafts- } \\
\text { bestandteilen/ Natur- } \\
\text { denkmalen } \\
\text { - Landschaftsschutzgebiet } \\
\text { (LRPL) }\end{array}$ \\
\hline
\end{tabular}

Abbildung 5 Auszug aus der Kriterienübersicht zur Bestimmung der Raumverträglichkeit von Rohstoffpotenzialflächen in der Region Rheinhessen-Nahe 1 Inklusive gesetzlicher Puffer und Bereiche mit Zustimmungspflicht. 2 Datengrundlagen noch nicht vorliegend. 3 Soweit aus den sich derzeit in Bearbeitung befindlichen Hinweiskarten des Landesamts für Geologie und Bergbau Rheinland-Pfalz und sonstigen Fachdaten räumlich konkret ableitbar. 4 Sofern keine Voreinschätzung/Einzelfallprüfung vorliegt, können Rohstoffpotenzialflächen weiterhin für die langfristige Rohstoffsicherung in Frage kommen. Sobald eine Voreinschätzung/Einzelfallprüfung zu einem positiven Ergebnis führt, können die im Plan gekennzeichneten Flächen gegebenenfalls wieder als Vorrangflächen für den Abbau festgelegt werden. 5 Die Biotope nach § 30 BNatSchG sind per se gesetzlich geschützt. Es gilt ein Beeinträchtigungsverbot. Jedoch muss ein einzelnes Biotop nach § 30 BNatSchG in einem größeren Rohstoffgebiet nicht zwingend zum Ausschluss des ganzen Gebietes führen. Erst wenn eine höhere Dichte solcher Biotope vorliegt, ist es mit großer Sicherheit nicht mehr möglich, einen Rohstoffabbau umzusetzen. Die Notwendigkeit einer möglichen Befreiung bleibt hiervon so oder so unberührt. Biotope nach $\S 30 \mathrm{BNatSchG}$ sind auf regionalplanerischer Ebene nicht abwägbar. Es muss eine Befreiung in Aussicht gestellt werden, d.h. im Rahmen einer Einzelfallentscheidung kann nach § 30 Abs. 3 BNatSchG von einem Beeinträchtigungsverbot auf Antrag eine Ausnahme zugelassen werden. Quelle: Eigene, gekürzte Darstellung auf der Basis von PGRN (2015a: 61ff.) 


\subsection{Methodik zur Vorbereitung der raumordnerischen Abwägung}

Ziel der raumordnerischen Abwägung ist es, Rohstoffpotenzialflächen entsprechend ihrer Eignung sowie des erwartbaren Raumwiderstands zu priorisieren und Flächenkontingente für die kurz- bis mittelfristige Rohstoffgewinnung sowie eine langfristige Rohstoffsicherung zu unterscheiden. Über eine nachvollziehbare Abwägung lässt sich die Auswahl geeigneter raumordnerischer Instrumente begründen.

Die raumordnerische Abwägung muss dem aktuellen Stand von Wissenschaft und Praxis entsprechen. Die Ergebnisse der raumordnerischen Abwägung sollen auch für Dritte nachvollziehbare Ergebnisse liefern. Vor allem bei besonderen Nutzungskonkurrenzen und Konfliktsituationen empfiehlt sich eine ausführliche Darlegung des Abwägungsprozesses (vgl. PGRN 2015a; Regionale Planungsgemeinschaft Havelland-Fläming 2015; BMVI 2017: 67 ff.; Region Hannover 2017). So wurde der Abwägungsprozess im Regionalplan Havelland-Fläming 2020 auf Basis eines Kriteriensets, das Restriktionen berücksichtigt, strukturiert: Es wird Schritt für Schritt nachvollziehbar darüber entschieden, „ob eine Fläche weiterhin als Vorranggebiet oder nur noch als Vorbehaltsgebiet fortgeprüft wird bzw. ob Flächen teilweise durch Flächenabzug bzw. vollständig entfallen" (Regionale Planungsgemeinschaft HavellandFläming 2015: 1008).

Bei komplexen Sachlagen, uneinheitlichen Daten und vielfältigen Wechselwirkungen oder kumulativen Wirkungen verbinden sich mit der Umsetzung dieses Anspruchs durchaus besondere Herausforderungen. Neben den erforderlichen Grundlagendaten zu den Rohstoffpotenzialflächen und den betroffenen Schutzgütern sollte die Abwägung deshalb auf der Basis einer kohärenten methodischen Vorgehensweise vorgenommen werden. Diese besteht im Kern aus einer Verknüpfung des Raumwiderstands, der sich durch die potenzielle Betroffenheit der Schutzgüter begründet, mit den bewerteten Rohstoffpotenzialflächen, also der Eignung oder Bauwürdigkeit der Rohstoffvorkommen. Darüber werden die Flächenkontingente priorisiert. Diese Priorisierung führt über die Bedarfsermittlung für die Rohstoffgewinnung zu einer Differenzierung des Flächenkontingents von Rohstoffgewinnung und langfristiger Rohstoffsicherung. Sie begründet somit die Zuordnung von Flächenkontingenten zu den raumordnerischen Instrumenten.

Spezifische Lösungen zeigen Regionalpläne, die eine Vorbelastung von Teilräumen bzw. kumulative Wirkungen in den Abwägungsprozess einbeziehen. Kumulative Effekte bezeichnen Wirkungen durch eine Vielzahl von Abbaustellen in räumlicher Nähe, die über die Summe der Auswirkungen von Einzelstandorten deutlich hinausgehen und eine Transformation von Teilräumen zur Folge haben können. Ein Beispiel ist die Region Hannover, die im Regionalen Raumordnungsprogramm die Wirkungen räumlicher Konzentrationen von Abbauflächen insbesondere in Teilräumen mit relativ hoher Bevölkerungs- und Siedlungsdichte berücksichtigt (Region Hannover 2017: 184). Auf dieser Grundlage wurde ein Planungskonzept zur räumlichen Abgrenzung für „Gebiete mit Ausschlusswirkung für Rohstoffgewinnung" in erheblich durch den Rohstoffabbau vorbelasteten Teilräumen erarbeitet.

Die unterschiedlichen Aspekte der raumordnerischen Abwägung lassen sich im Kontext einer zeitlichen und räumlichen Steuerung der Rohstoffsicherung zueinander in Relation setzen. Dies veranschaulicht Abbildung 6, wobei zunächst lediglich die wesentlichen Kategorien bzw. Grundausrichtungen der raumordnerischen Festlegungen berücksichtigt werden. Die Ausgestaltung der methodischen Bausteine und Verknüpfungen wie auch die Ausdifferenzierung und Zuteilung zu den raumordnerischen Instrumenten müssen sich an den spezifischen Fragestellungen und Rahmenbedingungen in der Region orientieren.

\subsection{Ausgestaltung der raumordnerischen Instrumente}

$\S 7$ ROG regelt die möglichen raumordnerischen Festlegungen für die Flächenvorsorge. Diese umfassen

- Vorranggebiete, die Nutzungen ausschließen, soweit diese mit den vorrangigen Funktionen oder Nutzungen nicht vereinbar sind. Vorranggebiete haben Zielcharakter und sind keiner weiteren Abwägung im Rahmen nachgeordneter Planungen zugänglich. Eine Überlagerung von Vorranggebieten ist möglich, sofern hieraus keine Zielkonflikte entstehen.

- Vorbehaltsgebiete, denen bei der Abwägung mit konkurrierenden raumbedeutsamen Nutzungen besonderes Gewicht beizumessen ist. Vorbehaltsgebiete haben Grundsatzcharakter. Hier bestehen Abwägungsspielräume.

- Eignungsgebiete, in denen bestimmte raumbedeutsame Maßnahmen oder Nutzungen, die städtebaulich nach $\S 35$ BauGB $^{11}$ zu beurteilen sind, anderen raumbedeutsamen Belangen nicht entgegenstehen. Sie schließen die betreffenden Maßnahmen oder Nutzungen andernorts aus.

Die Rohstoffsicherung wird auf der Ebene der Regionalplanung überwiegend über die Festlegung des Vorrangs und des Vorbehalts vorgenommen (vgl. SGD 2008: 19f.). Die Ausgestaltung der Instrumente zeigt deutliche Unterschiede zwischen den Regionalplänen. Dies bezieht sich beispielsweise auf eine räumliche und zeitliche Steuerung und somit auf eine Differenzierung von kurz- bis mittelfristiger Roh-

\footnotetext{
11 Baugesetzbuch in der Fassung der Bekanntmachung vom 3. Novem-
} ber 2017. 


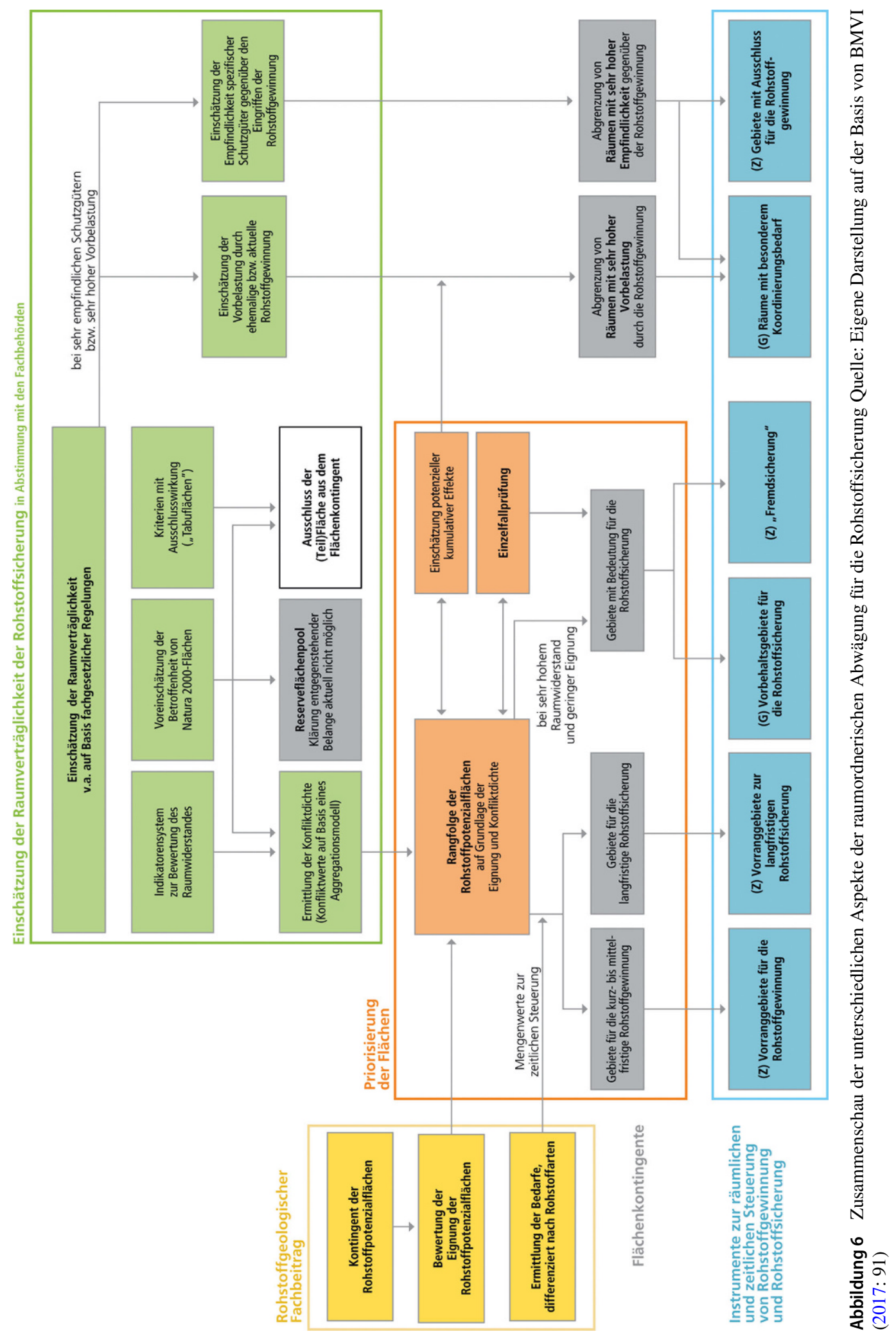


stoffgewinnung und langfristiger Rohstoffsicherung. Auch die Zeiträume der Festlegungen reichen von der Laufzeit der Regionalpläne mit etwa 15 Jahren bis zu einer unbefristeten langfristigen Rohstoffsicherung.

In einigen Regionalplänen werden Vorranggebiete (mit der Wirkung von Eignungsgebieten) mit einer Ausschlusswirkung für die Rohstoffgewinnung außerhalb der Gebiete verknüpft (z. B. im Regionalplan Düsseldorf; Bezirksregierung Düsseldorf 2016: 164). In Nordrhein-Westfalen wird diese Vorgehensweise bereits auf der Ebene der Landesplanung als Ziel formuliert, nämlich, dass in ,den Regionalplänen [...] Bereiche für die Sicherung und den Abbau oberflächennaher Bodenschätze für nichtenergetische Rohstoffe als Vorranggebiete mit der Wirkung von Eignungsgebieten festzulegen [sind]" (Landesregierung Nordrhein-Westfalen 2017: 98). In der Region Hannover bezieht sich der Ausschluss auf Teilräume mit Vorbelastungen durch eine hohe Dichte von Abbaustandorten (Region Hannover 2017: 184 ff.). Die Abbautätigkeit wird so auf bestimmte Bereiche konzentriert.

Eine Bündelungsfunktion kann bereits auf Landesebene über eine Festlegung von Konzentrationszonen erfolgen. Diesen Ansatz verfolgt beispielsweise der Landesentwicklungsplan Schleswig-Holstein, der über einen Grundsatz festlegt, dass ,der Abbau oberflächennaher Rohstoffe [...] landseitig vorrangig in Schwerpunkträumen erfolgen [soll]“. Diese Schwerpunkträume sind in den Regionalplänen „durch die Ausweisung von Vorrang- und Vorbehaltsgebieten für die Rohstoffsicherung [...] unter Abwägung mit konkurrierenden Flächenansprüchen zu konkretisieren“" (Innenministerium des Landes Schleswig-Holstein 2010: 81).

Auf regionaler Ebene kann eine verbindliche zeitliche Steuerung mit Vorranggebieten für die langfristige Rohstoffsicherung vorgenommen werden, wobei sich in diesen Gebieten die Rohstoffgewinnung explizit für den Geltungszeitraum des Regionalplans ausschließen lässt. Festlegungen dazu trifft beispielsweise der Regionale Raumordnungsplan Rheinhessen-Nahe (PGRN 2015b: 63): „Z 93: In den Vorranggebieten für die langfristige Rohstoffsicherung hat die Sicherung der oberflächennahen Rohstofflagerstätten Vorrang vor anderen Raumnutzungsansprüchen, die einem möglichen, zukünftigen Abbau der Rohstofflagerstätte entgegenstehen können. Die Vorranggebiete kommen für einen Rohstoffabbau innerhalb der Laufzeit des regionalen Raumordnungsplans nicht in Betracht.“ Darüber hinaus kommen auch explizit Ausschlussgebiete zum Einsatz, z. B. in Baden-Württemberg. Hier besteht die Möglichkeit, Ausschlussgebiete festzulegen, die eine Nutzung wie den Rohstoffabbau auf bestimmten Flächen verhindern (Regionalverband Hochrhein-Bodensee 2005: 50f.; SGD 2008: 48). Die Ausschlussgebiete dienen damit nicht der Rohstoffsicherung, vielmehr einer räumlichen Steuerung der Rohstoffgewinnung. Ausschlussgebiete werden in der Re- gel mit Vorrang- und Vorbehaltsgebieten zur Rohstoffsicherung kombiniert.

Eine Überlagerung mit anderen Vorrängen kann in Bezug auf Vorranggebiete zur Rohstoffsicherung dann sinnvoll sein, wenn einerseits Zwischennutzungen zugelassen werden sollen (z. B. Windkraftnutzung) oder andererseits damit bereits die Folgenutzung bestimmt wird.

Rohstoffgewinnung steht oftmals in direkter Konkurrenz $\mathrm{zu}$ anderen Raumnutzungen oder Raumfunktionen. Hieraus und auch aufgrund einer oftmals nicht gesicherten Datenlage entstehen schwierige Konfliktsituationen, die eines intensiveren Dialog- und Planungsprozesses bedürfen. Das Landesentwicklungsprogramm Thüringen führt eine neue Gebietskategorie ein: die „Räume mit besonderem Koordinationsbedarf" (TMBLV 2014: $108 \mathrm{ff}$.). Hierunter fallen Bereiche mit Rohstoffgruppen, die eine besondere Bedeutung oder ein begrenztes Potenzial besitzen, sowie Räume, die durch eine intensive Überlagerung von Rohstoffsicherungsgebieten mit konkurrierenden Raumnutzungsansprüchen gekennzeichnet sind. Bei hohen Raumwiderständen gegenüber einer Rohstoffsicherung bietet es sich unter Umständen an, Gebiete mit besonderer und meist langfristiger Bedeutung für die Rohstoffsicherung über eine Fremdsicherung durch andere raumordnerische Kategorien zu sichern, beispielsweise über Vorrangflächen zu Landwirtschaft und Hochwasserschutz, für Wald- und Naturschutz oder zur Freiraumsicherung. In Verbindung mit einem Überbauungsverbot in diesen Gebieten wird zumindest das Ziel erreicht, dass sich keine persistenten, der Rohstoffgewinnung entgegenstehenden Nutzungen etablieren können.

In Abhängigkeit von landesplanerischen Vorgaben und regionalen Aufgaben zur Rohstoffsicherung kann somit eine Bandbreite raumordnerischer Instrumente zum Einsatz kommen (vgl. Tabelle 3). Sinnvoll ist eine räumliche und zeitliche Steuerung im Sinne einer Differenzierung von kurz- bis mittelfristiger Rohstoffgewinnung und langfristiger bzw. vorsorgender Rohstoffsicherung.

Weitergehende Regelungen sollten über Grundsätze oder Ziele in die Landes- und Regionalplanung eingebettet werden (BMVI 2017: 93). Hierzu können Regelungen gehören

- zu Substitution und Recycling von Rohstoffen,

- zur Priorisierung einer Erweiterung bestehender Abbaustellen gegenüber dem Neuaufschluss bislang unverritzter Flächen,

- zur Minimierung der Eingriffe in den Naturhaushalt,

- zu einem möglichst vollständigen Abbau von Lagerstätten,

- zur Vermeidung oder Minderung nachteiliger Auswirkungen auf andere Schutzgüter,

- zur Bewältigung von Nutzungskonkurrenzen im Sinne einer nachhaltigen Raumentwicklung, 
Tabelle 3 Zusammenschau der unterschiedlichen raumordnerischen Instrumente zur räumlichen und zeitlichen Steuerung von Rohstoffgewinnung und (vorsorgender) Rohstoffsicherung auf Landes- und Regionalplanungsebene

\begin{tabular}{lll}
\hline Instrument & Aufsuchung und Gewinnung von Rohstoffen & Vorsorgende Rohstoffsicherung \\
Vorranggebiet & - Letztabwägung auf Grundlage der vorliegenden & - Letztabwägung auf Grundlage der vorliegenden \\
& Informationen erforderlich & Informationen erforderlich \\
& - Bedarfsorientierte Ausweisung & - Bedarfsunabhängige Ausweisung \\
- Planungszeitraum: mindestens 25 Jahre (im Interes- & - Planungszeitraum: unbefristet \\
& se der Rohstoffwirtschaft) & - Gegebenenfalls mit explizitem Ausschluss der Roh- \\
& - Gegebenenfalls in Verbindung mit einer Auswei- & stoffgewinnung innerhalb des Sicherungsgebietes \\
sung als Eignungsgebiet mit Ausschlusswirkung für & - Gegebenenfalls Überlagerung von Vorrängen der \\
einen Rohstoffabbau außerhalb der Vorranggebiete. & langfristigen Rohstoffsicherung mit anderen nicht \\
Die Ausschlusswirkung kann sich auf die gesamte & entgegenstehenden Vorrängen. \\
\hline Region oder auf definierte Teilräume beziehen Sie &
\end{tabular}

Vorbehaltsgebiet

Fremdsicherung über Festlegungen zu anderen Raumnutzungen oder Raumfunktionen

\section{Schwerpunkträume}

Räume mit besonderem Koordinationsbedarf

Gebiete mit einem Ausschluss für die Rohstoffgewinnung
Region oder auf definierte Teilräume beziehen. Sie kann zudem einzelne Rohstoffarten betreffen.

Letztabwägung aufgrund entgegenstehender Umweltbelange, fehlender Informationen zur Rohstoffgeologie bzw. zu Umweltaspekten oder aufgrund von Rohstofflagerstätten mit geringer Eignung (aktuell noch) nicht möglich; Berücksichtigung des Belangs der Rohstoffsicherung bei allen nachgeordneten Verfahren.

- Festlegung mit Zielcharakter bei im Rahmen der Abwägung nicht lösbaren Konfliktsituationen, um Nutzungen, die perspektivisch einer Rohstoffgewinnung entgegenstehen, auszuschließen. Eine Fremdsicherung kann in erster Linie über vorrangige Festlegungen zu Landwirtschaft, Hochwasser-, Wald- und Naturschutz oder zur Freiraumsicherung erzielt werden. Hier steht Siedlungsentwicklung der vorrangigen Raumnutzung entgegen.

- Gegebenenfalls mit Ausschluss der Rohstoffgewinnung innerhalb der festgelegten Gebiete für die Laufzeit des Regionalplans.

Über die Festlegung von Konzentrationszonen bzw. Schwerpunkträumen erfolgt eine Bündelung der Rohstoffgewinnung. Der Abbau oberflächennaher Rohstoffe soll vorrangig in bestimmten Bereichen erfolgen.

Sonstige Erfordernisse zum Umgang mit Teilräumen, die aufgrund

$\rightarrow$ aktueller Vorbelastungen durch eine hohe Dichte von Abbauflächen und/oder großflächigem Rohstoffabbau einer besonderen Belastung bzw. Transformation ausgesetzt sind.

$\rightarrow$ geplanter Vorhaben zur Rohstoffgewinnung und erwarteter (kumulativer) Auswirkungen ein hohes Konfliktpotenzial aufweisen.

$\rightarrow$ spezifischer und (über-)regional bedeutsamer Rohstoffvorkommen einer besonderen Sicherung bedürfen. Hiermit kann ein Impuls für nachgeordnete Verfahren und dialogorientierte Prozesse verbunden und bereits auf der Ebene der Regionalplanung als Auftrag formuliert werden.

Festlegung mit Zielcharakter für Gebiete, in denen eine Rohstoffgewinnung für die Laufzeit des Regionalplans ausgeschlossen wird. Ausschlussgebiete sichern somit Räume mit empfindlichen Schutzgütern oder Bereiche mit hoher Vorbelastung. Eine vorrangige Nutzung oder Funktion ist festzulegen.

Quelle: Eigene, veränderte Darstellung auf der Basis von BMVI (2017: 94)

- zu Zwischennutzungen und einer Priorisierung sich überlagernder Vorränge,

- zu Renaturierung/Rekultivierung (bereits während des Abbaus) und zu Folgenutzungen nach dem Abbau.

\subsection{Umgang mit Folgenutzungen auf der Ebene der Regionalplanung}

Im Bereich der Folgenutzungen differieren die Festlegungen in den Regionalplänen erheblich: von allgemeinen Grundsätzen bis hin zu konkreten Zielen. Wenn großflächiger Rohstoffabbau oder eine hohe Dichte an kleineren Abbaustandorten absehbar zu einer großräumigen Transformation von Teilräumen und Landschaften führen wird, kann es durchaus sinnvoll sein, frühzeitig regionalplanerische Festlegungen $\mathrm{zu}$ Folgenutzungen $\mathrm{zu}$ treffen. Die Regelungstiefe kann bis hin zu standortbezogenen Zuweisungen reichen, wie beispielsweise in der Region DonauIller, wo den im Regionalplan ausgewiesenen Vorrang- und Vorbehaltsgebieten der Rohstoffsicherung jeweils konkrete Folgenutzungen zugewiesen werden (Regionalverband Donau-Iller 2006). Durch eine verbindliche Steuerung kann die Akzeptanz für Abbauvorhaben in der Region erhöht werden. Gleichzeitig sind frühe, sehr konkrete Festlegungen zu Folgenutzungen mit Unsicherheiten behaftet, zumal der Abbau oftmals Jahrzehnte in Anspruch nimmt. Sie bedeuten durchaus eine Einschränkung der Gestaltungsspielräume, um auf veränderte regionale Rahmenbedingungen zu reagieren. Bei hohen Nutzungskonkurrenzen 
und Konfliktpotenzialen ist es zudem ratsam, eigenständige Konzepte zu Folgenutzungen zu erarbeiten. Hier bietet sich eine Beteiligung regionaler und lokaler Akteure sowie der Bevölkerung an, um die Folgenutzungen am Bedarf der Bevölkerung vor Ort, an kommunalen Rahmenbedingungen sowie einem potenziellen regionalen Mehrwert auszurichten (vgl. PGRN 2015a). Auch die Ministerkonferenz für Raumordnung empfiehlt die Erstellung und Umsetzung regionaler Konzepte zur Rekultivierung von Abbaustandorten und zur Etablierung integrierter Folgenutzungen (MKRO 2016: 26). Auch wenn die Regelung auf nachgeordneter Ebene meist eine höhere Passgenauigkeit der Folgenutzungen ermöglicht, empfiehlt es sich, zumindest grundsätzliche regionalplanerische Festlegungen zu treffen, um Rahmenbedingungen für eine an den Bedarfen der Region orientierten Form der Wiedernutzbarmachung zu schaffen.

Das Land Nordrhein-Westfalen gibt so in einem eigenen Ziel zu Folgenutzungen den Regionalplanungsträgern vor, dass „Flächen, die dem Abbau oberflächennaher Bodenschätze dienen, [..] abschnittsweise und zeitnah zu rekultivieren bzw. wiedernutzbar zu machen [sind]. In den Regionalplänen ist die Nachfolgenutzung für diese Flächen zeichnerisch festzulegen" (Landesregierung Nordrhein-Westfalen 2017: 9.2-4 Ziel Nachfolgenutzung). Was von vielen Regionsvertretern im Rahmen der Befragung angemerkt wurde, ist die Überlagerungsmöglichkeit von Vorranggebieten, um Zielkonflikte zu entschärfen bzw. die grundsätzliche Ausrichtung der Folgenutzungen zu definieren, z. B. in Bezug auf Windenergienutzung oder Naturschutz.

Die unterschiedlichen Herangehensweisen zeigen, dass im Hinblick auf die spezifische regionale Situation entschieden werden muss, ob der Schwerpunkt auf einer frühzeitigen, weitreichenden und konkreten Regelung zu Folgenutzungen liegen sollte oder darauf, grundsätzliche Erfordernisse und Ausrichtungen festzulegen, um Rahmenbedingungen für nachgeordnete Planungen zu formulieren. In jedem Fall sind gerade im Kontext von Folgenutzungen kumulative Effekte zu berücksichtigen und auf eine Einbettung der Rohstoffgewinnung in eine integrative und nachhaltige Raumentwicklung hinzuwirken. Abbaubereiche, die im Nachgang zur Rohstoffgewinnung hochwertig genutzt werden, können sich zu attraktiven Erholungsgebieten und/ oder wichtigen Lebensräumen seltener Tier- und Pflanzenarten entwickeln. Daher gilt es, die Chancen, die sich aus dem Abbau mineralischer Bodenschätze ergeben, gemeinsam auszuloten und in Bezug auf den Detaillierungsgrad regionalplanerischer Festlegungen eine Verständigung zwischen den Akteuren zu erzielen.

\subsection{Informelle Strategien auf der Ebene der Landes- und Regionalplanung}

Informelle Konzepte und Planungsprozesse dienen nicht nur einer Konkretisierung von Rekultivierungen und Folgenutzungen. Auch die Rohstoffsicherung kann im Vorfeld der Regionalplanung durch informelle Konzepte und Prozesse grundsätzlich vorbereitet bzw. flankiert werden. Auf den Ebenen der Länder und Regionen liegen bereits vielversprechende Erfahrungen vor: Hierzu zählen etwa Rohstoffsicherungskonzepte, teilräumliche (integrierte) Entwicklungskonzepte oder auch dialogorientierte Formen der sektorübergreifenden behördlichen Zusammenarbeit bzw. der Akteur- und Öffentlichkeitsbeteiligung. Rohstoffsicherungskonzepte können einen Grundkonsens herstellen und einen Orientierungsrahmen für den formalen Planungsprozess schaffen. Beispiele sind die Rohstoffsicherungskonzepte in Baden-Württemberg (Wirtschaftsministerium Baden-Württemberg 2004) oder in Hessen (HMULV 2007).

Die Regionalen Planungsverbände im Regierungsbezirk Unterfranken (Region Bayerischer Untermain, Region Würzburg, Region Main-Rhön) konnten auf der Basis von Rohstoffkonzepten bereits im Vorfeld zur formalen Regionalplanung Konfliktlösungen aufzeigen (BMVI 2017: 76). Im Pilotvorhaben der Planungsgemeinschaft RheinhessenNahe wurde ein Dialogverfahren durchgeführt, um zwischen den verschiedenen Interessengruppen wie Rohstoffverbänden, Unternehmen, Kommunen, Naturschutzverbänden, Fachbehörden und Bürgerinitiativen zu vermitteln und das Verfahren der formalen Abwägung vorzubereiten (PGRN 2015a: 2f.). Der Landkreis Nienburg/Weser hat mit einem Bodenabbauleitplan, einem Rahmenkonzept für einen Kompensationsflächenpool sowie mit der Abstimmung zwischen agrarstruktureller Entwicklungsplanung und Rohstoffgewinnung einen integrativen Ansatz verfolgt, unter anderem um Nutzungskonflikte zu minimieren, teilräumliche Belastungen auszugleichen und eine Koordination von Raumnutzungen zu fördern (Landkreis Nienburg/Weser 2003). In Hessen hat sich beispielsweise das „Dialogforum Rohstoffsicherheit“ formiert - ein Gesprächskreis, der sich aus Vertretern der betroffenen Wirtschaft und der zuständigen Behörden zusammensetzt und sich unter anderem an der Erstellung des „Rohstoffsicherungskonzepts Hessen“ beteiligt hat. Das Dialogforum beschäftigt sich mit aktuellen Herausforderungen von Rohstoffsicherung und Rohstoffabbau im Kontext von Naturschutz (Natura 2000) und Raumordnung. ${ }^{12}$

\footnotetext{
12 http://www.umweltallianz.de/de/rohstoffsicherheit.html (15.03.2018).
} 


\section{Fazit}

Da es keine Rohstofffachplanung gibt, übernimmt die Raumordnung auf der Basis des Raumordnungsgesetzes eine zentrale Aufgabe bei der Rohstoffsicherung in Deutschland. Der Beitrag hat gezeigt, dass das gesetzliche Instrumentarium ausreicht, um diesen Auftrag auszufüllen. Die dargestellten methodischen Ansätze zur Bewältigung des Abwägungsprozesses wie auch zur Ausgestaltung der raumordnerischen Instrumente geben konkrete Hinweise, wie sowohl die Rohstoffgewinnung als auch die langfristige Rohstoffsicherung in die Landes- und Regionalplanung eingebettet werden können. Die Ansätze können den Ländern und Regionen als Orientierungsrahmen zur Weiterentwicklung der raumordnerischen Verankerung einer zukunftsfähigen Rohstoffsicherung dienen.

Um die Belange der Rohstoffsicherung im Rahmen der Raumordnung substanziell darstellen zu können, wird von den Akteuren der Raumordnung und der Rohstoffgewinnung zu Recht gefordert, dass sich die Grundlagendaten in ihrer Qualität an den Grundlagen anderer, oftmals konkurrierender Fachplanungen messen lassen müssen. Hierzu zählt insbesondere die Bewertung der Rohstoffpotenzialflächen anhand der Eignung. Nur so kann die Rohstoffsicherung im Rahmen der Abwägung das erforderliche Gewicht entfalten.

Der Abwägung kommt eine besondere Rolle zu. Der Beitrag legt dar, wie zentrale Aspekte der Abwägung zusammenwirken, sodass die unterschiedlichen Flächenkontingente entsprechend ihrer Eignung und unter Berücksichtigung entgegenstehender Belange nachvollziehbar den raumordnerischen Instrumenten zugeordnet werden können.

In den Ländern und Regionen kommt eine Vielfalt an Instrumenten zum Einsatz. Der Beitrag ordnet diese in eine Systematik ein, die beiden Teilaufgaben gerecht wird. Gleichwohl sind der Raumordnung auch klare Grenzen gesetzt. Dies zeigt sich unter anderem im Umgang mit der Natura-2000-Kulisse. Zudem unterliegt die Rohstoffsicherung - wie alle anderen Raumnutzungen und Raumfunktionen - der Abwägung, mithin der politischen Entscheidung.

In Regionen, in denen erhebliche Flächenkonkurrenzen und Widerstände die Rohstoffsicherung erschweren, kann es sich insbesondere lohnen, die unterschiedlichen Ansätze von Rohstoffgewinnung und vorsorgender Rohstoffsicherung über kommunikative bzw. dialogische Verfahren transparenter zu kommunizieren.

\section{Literatur}

agl Hartz $\bullet$ Saad $\bullet$ Wendl (2016): Rohstoffsicherungsplanung. Begleitprojekt zum neuen Regionalen Raumordnungsplan Region Trier.
Bericht zum Abschluss des Projekt-Moduls 1 (Konfliktanalyse). Saarbrücken.

Bezirksregierung Düsseldorf (2016): Regionalplan Düsseldorf, 2. Entwurf, Stand: Juni 2016. Düsseldorf. https://www.brd.nrw.de/ planen_bauen/regionalplan/rpd_2e_062016.html (15.03.2018).

BGR - Bundesanstalt für Geowissenschaften und Rohstoffe (2014): Deutschland - Rohstoffsituation 2013. Hannover.

BGR - Bundesanstalt für Geowissenschaften und Rohstoffe (2015): Deutschland - Rohstoffsituation 2014. Hannover.

BGR - Bundesanstalt für Geowissenschaften und Rohstoffe (2017): Deutschland - Rohstoffsituation 2016. Hannover.

BMVI - Bundesministerium für Verkehr und digitale Infrastruktur (Hrsg.) (2017): Mittel- und langfristige Sicherung mineralischer Rohstoffe in der landesweiten Raumplanung und in der Regionalplanung. Berlin. = MORO Praxis 9/2017.

BMWi - Bundesministerium für Wirtschaft und Technologie (2010): Rohstoffstrategie der Bundesregierung. Sicherung einer nachhaltigen Rohstoffversorgung Deutschlands mit nicht-energetischen mineralischen Rohstoffen. Berlin.

Bundesverband Baustoffe - Steine und Erden (2010): Leitfaden Raumordnung. Berlin.

Bundesverband Baustoffe - Steine und Erden (2015): Mineralische Bauabfälle. Monitoring 2012. Bericht zum Aufkommen und zum Verbleib mineralischer Bauabfälle im Jahr 2012. Berlin.

Bundesverband Baustoffe - Steine und Erden (2016): Die Nachfrage nach Primär- und Sekundärrohstoffen der Steine-und-Erden-Industrie bis 2035 in Deutschland. Berlin.

DERA - Deutsche Rohstoffagentur in der Bundesanstalt für Geowissenschaften und Rohstoffe (2014): DERA-Rohstoffliste 2014. Berlin. = DERA-Rohstoffinformationen 24.

Düppenbecker, A.; Greiving, S. (1999): Die Auswirkungen der Fauna-Flora-Habitat-Richtlinie und der Vogelschutzrichtlinie auf die Bauleitplanung. In: Umwelt- und Planungsrecht 19, 5, 173-178.

Erdmann, L.; Behrendt, S.; Feil, M. (2011): Kritische Rohstoffe für Deutschland. Identifikation aus Sicht deutscher Unternehmen wirtschaftlich bedeutsamer mineralischer Rohstoffe, deren Versorgungslage sich mittel- bis langfristig als kritisch erweisen könnte. Kurzfassung. Berlin.

Europäische Kommission (2011): Grundstoffmärkte und Rohstoffe: Herausforderungen und Lösungsansätze. Mitteilung der Kommission an das Europäische Parlament, den Rat, den europäischen Wirtschafts- und Sozialausschuss und den Ausschuss der Regionen. KOM(2011) 25 endgültig. Brüssel.

HMULV - Hessisches Ministerium für Umwelt, ländlichen Raum und Verbraucherschutz (2007): Rohstoffsicherung in Hessen. Wiesbaden.

Innenministerium des Landes Schleswig-Holstein (2010): Landesentwicklungsplan Schleswig-Holstein 2010. Kiel.

Jeschke, A. (2004): Raumplanung als vorsorgendes Instrument im Küstenschutzmanagement. Masterarbeit, Carl von Ossietzky Universität Oldenburg. http://www.ikzm-strategie.de/dokumente/ jeschke_2004_raumplanung_kuestenschutz_ns.pdf (13.03.2018).

Kommission der Europäischen Gemeinschaften (2008): Die Rohstoffinitiative - Sicherung der Versorgung Europas mit den für Wachstum und Beschäftigung notwendigen Gütern. Mitteilung der Kommission an das europäische Parlament und den Rat. KOM(2008) 699 endgültig. Brüssel.

Krenzke, S. (2010): Aufbereitung und Verwertung von Bauabfällen in Baden-Württemberg im Kontext der neuen Abfallgesetzgebung. In: Statistisches Monatsheft Baden-Württemberg 9, 44-47.

Krumm, R. (2014): Nicht-energetische Rohstoffe: Datenlage in Deutschland und Baden-Württemberg sowie rohstoffökonomische Überlegungen zur Schließung von Datenlücken. Tübingen. = IAW Policy Report 11 .

Kukuk, P. (1960): Geologie, Mineralogie und Lagerstättenlehre. Berlin.

Landesregierung Nordrhein-Westfalen (2017): Landesentwicklungsplan Nordrhein-Westfalen (LEP NRW). Düsseldorf. 
Landkreis Nienburg/Weser (2003): Regionales Raumordnungsprogramm (2003) Landkreis Nienburg/Weser. Nienburg an der Weser.

LGRB - Landesamt für Geologie, Rohstoffe und Bergbau BadenWürttemberg (2013): Rohstoffbericht Baden-Württemberg 2012/ 2013. Bedarf, Gewinnung und Sicherung von mineralischen Rohstoffen. Dritter Landesrohstoffbericht. Freiburg. = LGRBInformationen 27.

Matthes, S. (2001): Mineralogie. Eine Einführung in die spezielle Mineralogie, Petrologie und Lagerstättenkunde. Berlin/Heidelberg.

MKRO - Ministerkonferenz für Raumordnung (2016): Leitbilder und Handlungsstrategien für die Raumentwicklung in Deutschland 2016. Verabschiedet von der Ministerkonferenz für Raumordnung am 9. März 2016. Berlin.

PGRN - Planungsgemeinschaft Rheinhessen-Nahe (2015a): Regionale Rohstoffsicherung - Ziele, Erfahrungen und Ergebnisse des Pilotprojektes „Nachhaltiges Rohstoffsicherungskonzept“ im Zuge der Neuaufstellung des Regionalplans Rheinhessen-Nahe. Mainz.

PGRN - Planungsgemeinschaft Rheinhessen-Nahe (2015b): Regionaler Raumordnungsplan Rheinhessen-Nahe 2014. Mainz.

Region Hannover (2017): Regionales Raumordnungsprogramm Region Hannover 2016. Hannover.

Regionale Planungsgemeinschaft Havelland-Fläming (2015): Regionalplan Havelland-Fläming 2020. Teltow.

Regionalverband Donau-Iller (2006): Regionalplan (3.Teilfortschreibung) - Gewinnung und Sicherung von Bodenschätzen. Ulm.

Regionalverband Hochrhein-Bodensee (2005): Teilregionalplan Oberflächennahe Rohstoffe für die Region Hochrhein-Bodensee. Waldshut-Tiengen.

Schröder, B.; Vulpius, N. (2013): Anforderungen an die Rohstoffsicherung und deren unterschiedliche Umsetzung in der Landespla- nung. In: Schulz, M.; Schröder, B.; Vulpius, N. (Hrsg.): Rohstoffsicherung - kein alter Hut! Iffezheim, 4-7.

Schröder, B.; Vulpius, N. (2016): Rohstoffsicherung und Landesplanung. Anforderungen an die Umsetzung. In: Planerin 4, 66-68.

Schröder, N. (2017): Raumplanerische Rohstoffsicherung. Politische Orientierungen und rechtliche Grundlagen. In: Raumplanung 190, 2, 63-67.

SGD - Staatliche Geologische Dienste der Bundesrepublik Deutschland (2008): Rohstoffsicherung in der Bundesrepublik Deutschland - Zustandsbericht. http://www.infogeo.de/dokumente/down load_pool/rohstoffsicherung_2008.pdf (15.03.2018).

Spiekermann, J. (2009): Klimafolgenbewältigung in der Region Unterweser. Eine Betrachtung aus Sicht der Raumplanung. Diplomarbeit, Technische Universität Dortmund.

TMBLV - Thüringer Ministerium für Bau, Landesentwicklung und Verkehr (2014): Landesentwicklungsprogramm Thüringen 2025. Erfurt.

von Seht, H. (2010): Die regionalplanerische Steuerung des Abbaus nichtenergetischer Bodenschätze. In: Raumforschung und Raumordnung 68, 5, 419-431. doi: 10.1007/s13147-010-0056-0

Weber, F.; Jenal, C.; Kühne, O. (2017): Die Gewinnung mineralischer Rohstoffe als landschaftsästhetische Herausforderung. Eine Annäherung aus sozialkonstruktivistischer Perspektive. In: Kühne, O.; Megerle, H.; Weber, F. (Hrsg.): Landschaftsästhetik und Landschaftswandel. Wiesbaden, 245-266. doi: 10.1007/978-3658-15848-4_14

Wirtschaftsministerium Baden-Württemberg (2004): Rohstoffsicherungskonzept des Landes Baden-Württemberg. Stufe 2 „Nachhaltige Rohstoffsicherung“. Stuttgart. 